\title{
Solvability of an unsaturated porous media flow problem with thermomechanical interaction *
}

\author{
Bettina Albers ${ }^{\dagger} \quad$ Pavel Krejčí ${ }^{\ddagger} \quad$ Elisabetta Rocca ${ }^{\S}$
}

September 23, 2021

\begin{abstract}
A PDE system consisting of the momentum balance, mass balance, and energy balance equations for displacement, capillary pressure, and temperature as a model for unsaturated fluid flow in a porous viscoelastoplastic solid is shown to admit a solution under appropriate assumptions on the constitutive behavior. The problem involves two hysteresis operators accounting for plastic and capillary hysteresis.
\end{abstract}

\section{Introduction}

In a deformable porous solid filled with two immiscible fluids (water and air, say), two sources of hysteresis are observed: the solid itself is subject to irreversible plastic deformations, and the fluid flow exhibits capillary hysteresis which is often explained by the surface tension on the interfaces between the two fluids. A lot of works have been devoted to this phenomenon, see, e. g., [1, 2, 3, 4, 10, 11, 13. Mathematical analysis of various mechanical porous media models with capillary hysteresis and without temperature effects has been carried out in [6, 7, 22, 23. A PDE system for elastoplastic porous media flow with thermal interaction was derived in [5], but the existence of solutions was only proved for the isothermal case.

Here, we focus on the qualitative analysis of the model derived in [5], assuming in addition that the heat conductivity depends in a controlled way on the temperature similarly as in the phase transition model considered in [21]. Indeed, we borrow here some techniques employed in [21] and [5] in order to prove existence of a weak solution for the initial boundary value problem

*Supported by the GAČR Grant GA15-12227S and RVO: 67985840 (PK), and by the FP7-IDEAS-ERC-StG Grant \#256872 EntroPhase and GNAMPA (Gruppo Nazionale per l'Analisi Matematica, la Probabilità e le loro Applicazioni) of INdAM (Istituto Nazionale di Alta Matematica) (ER).

${ }^{\dagger}$ University of Duisburg-Essen, Geotechnical Engineering, D-45117 Essen, Germany, E-mail bettina.albers@uni-due.de.

¥Institute of Mathematics, Czech Academy of Sciences, Žitná 25, CZ-11567 Praha 1, Czech Republic, E-mail krejci@math.cas.cz.

$\S$ WIAS Berlin, Mohrenstr. 39, D-10117 Berlin, Germany, E-mail rocca@wias-berlin.de. 
associated with the PDE system coupling the momentum balance (cf. (1.4)), featuring, in particular, a thermal expansion term depending on the temperature field, with a mass balance (cf. (1.5)) ruling the evolution of the capillary pressure, and an energy balance (cf. (1.6)) displaying, in particular, quadratic dissipative terms on the right hand side.

The main mathematical difficulties are related to the low regularity of the temperature field mainly due to the presence of the high order dissipative terms in the internal energy balance. This is the reason why we need to employ here a key-estimate (cf. (5.3) ), already exploited in [9] and more recently in [21] for the analysis of non-isothermal phase transition models. Roughly speaking, since the test of the internal energy balance by the temperature $\theta$ is not allowed, we test by a suitable negative power of it and use the growth condition of the heat conductivity $\kappa$ in Hypothesis 3.1 (ii). Another key point in our proof is the $L^{\infty}$ estimate we get on the pressure which entails a bound in a proper negative Sobolev space for the time derivative of the absolute temperature, which turns out to be another fundamental ingredient in order to pass to the limit in our approximation scheme.

The structure of the paper is as follows. The model from [5] is briefly summarized in Section 1, In Section 2 we recall the definitions and main results of the theory of hysteresis operators that are used here. Section 3 contains the mathematical hypotheses and statements of the main results. In Section 4 we regularize the problem by adding a small parameter $\delta$ accounting for "micro-movements" and a large cut-off parameter $R$ to control the nonlinearities, and solve the regularized problem by the standard Faedo-Galerkin method. In Section 5 we let $\delta$ tend to 0 and $R$ to $\infty$ and prove that in the limit, we obtain a solution to the original problem.

\section{The model}

Consider a domain $\Omega \subset \mathbb{R}^{3}$ filled with a deformable solid matrix material with pores containing a mixture of liquid and gas. We state the balance laws in referential (Lagrangian) coordinates, assume the deformations small, and denote for $x \in \Omega$ and time $t \in[0, T]$

$$
\begin{aligned}
& u(x, t) \ldots \text { displacement vector of the referential particle } x \text { at time } t ; \\
& \varepsilon(x, t)=\nabla_{s} u(x, t) \ldots \text { linear strain tensor, }\left(\nabla_{s} u\right)_{i j}:=\frac{1}{2}\left(\frac{\partial u_{i}}{\partial x_{j}}+\frac{\partial u_{j}}{\partial x_{i}}\right) \\
& \sigma(x, t) \ldots \text { stress tensor; } \\
& p(x, t) \ldots \text { capillary pressure; } \\
& \theta(x, t) \ldots \text { absolute temperature; } \\
& A(x, t) \ldots \text { relative gas content. }
\end{aligned}
$$

For the stress $\sigma$ and gas content $A$ we assume the empirical constitutive relations

$$
\begin{aligned}
\sigma & =\mathbf{B} \varepsilon_{t}+P[\varepsilon]+\left(p-\beta\left(\theta-\theta_{c}\right)\right) \mathbf{1} \\
A & =G[p]
\end{aligned}
$$


where $P$ is a hysteresis operator describing the elastoplastic response of the solid, see Subsection 2.1. $\mathbf{B}$ is a constant symmetric positive definite fourth order viscosity tensor, $\beta \in \mathbb{R}$ is the relative solid-liquid thermal expansion coefficient, $\theta_{c}>0$ is a fixed referential temperature, $\mathbf{1}$ is the Kronecker tensor, and $G$ is a hysteresis operator as on Figure 1, see Subsection 2.2. We will see that both hysteresis operators $P$ and $G$ admit hysteresis potentials $V_{P}$ (clockwise) and $V_{G}$ (counterclockwise) and dissipation operators $D_{P}, D_{G}$ such that for all absolutely continuous inputs $\varepsilon, p$, the inequalities

$$
P[\varepsilon]: \varepsilon_{t}-V_{P}[\varepsilon]_{t}=\left\|D_{P}[\varepsilon]_{t}\right\|_{*}, \quad G[p]_{t} p-V_{G}[p]_{t}=\left|D_{G}[p]_{t}\right|
$$

hold almost everywhere, where $\|\cdot\|_{*}$ is a seminorm in $\mathbb{R}_{\mathrm{sym}}^{3 \times 3}$.

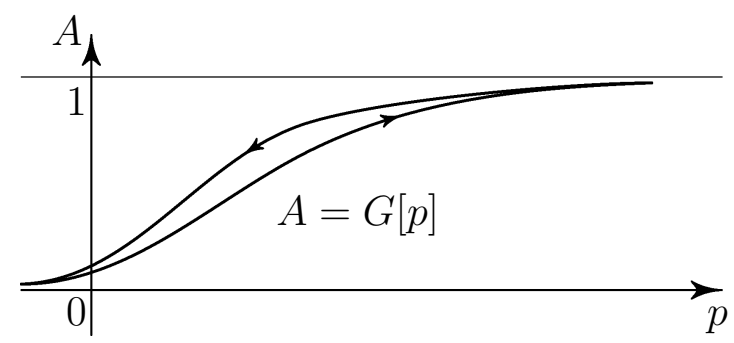

Figure 1: Pressure-saturation hysteresis diagram

We assume the heat conductivity $\kappa(\theta)$ depending on $\theta$, and as in [5], we obtain the system of momentum balance (1.4), mass balance (1.5), and energy balance equations (1.6) in the form

$$
\begin{aligned}
\rho_{S} u_{t t}= & \operatorname{div}\left(\mathbf{B} \nabla_{s} u_{t}+P\left[\nabla_{s} u\right]\right)+\nabla p-\beta \nabla \theta+g, \\
G[p]_{t}= & \operatorname{div} u_{t}+\frac{1}{\rho_{L}} \operatorname{div}(\mu(p) \nabla p), \\
c_{0} \theta_{t}= & \operatorname{div}(\kappa(\theta) \nabla \theta)+\left\|D_{P}\left[\nabla_{s} u\right]_{t}\right\|_{*}+\left|D_{G}[p]_{t}\right|+\mathbf{B} \nabla_{s} u_{t}: \nabla_{s} u_{t}+\frac{1}{\rho_{L}} \mu(p)|\nabla p|^{2} \\
& -\beta \theta \operatorname{div} u_{t},
\end{aligned}
$$

where $c_{0}>0$ is a constant specific heat, $\rho_{S}, \rho_{L}$ are the mass densities of the solid and liquid, respectively, $\mathbf{B}$ is a positive definite viscosity matrix, $\beta \in \mathbb{R}$ is the relative thermal expansion coefficient, and $g$ is a given volume force (gravity, e.g.).

We complement the system with initial conditions

$$
u(x, 0)=u^{0}(x), \quad u_{t}(x, 0)=u^{1}(x), \quad p(x, 0)=p^{0}(x), \quad \theta(x, 0)=\theta^{0}(x),
$$

and boundary conditions

$$
\left.\begin{array}{rl}
u & =0 \\
\mu(p) \nabla p \cdot n & =\gamma_{p}(x)\left(p^{*}-p\right) \\
\kappa(\theta) \nabla \theta \cdot n & =\gamma_{\theta}(x)\left(\theta^{*}-\theta\right)
\end{array}\right\} \text { on } \partial \Omega
$$

where $\gamma_{p}, \gamma_{\theta}: \partial \Omega \rightarrow[0, \infty)$ are given smooth functions. 


\section{Hysteresis operators}

We recall here the basic concepts of the theory of hysteresis operators that are needed in the sequel.

\subsection{The operator $P$}

In (1.1), $P$ stands for the elastoplastic part $\sigma^{e p}$ of the stress tensor $\sigma$. We proceed as in [18] and assume that $\sigma^{e p}$ can be represented as the sum $\sigma^{e p}=\sigma^{e}+\sigma^{p}$ of an elastic component $\sigma^{e}$ and plastic component $\sigma^{p}$. While $\sigma^{e}$ obeys the classical linear elasticity law

$$
\sigma^{e}=\mathbf{A}^{e} \varepsilon
$$

with a constant symmetric positive definite fourth order elasticity tensor $\mathbf{A}^{e}$, for the description of the behavior of $\sigma^{p}$, we split also the strain tensor $\varepsilon$ into the sum $\varepsilon=\varepsilon^{e}+\varepsilon^{p}$ of the elastic strain $\varepsilon^{e}$ and plastic strain $\varepsilon^{p}$, and assume

$$
\sigma^{p}=\mathbf{A}^{p} \varepsilon^{e}
$$

again with a constant symmetric positive definite fourth order elasticity tensor $\mathbf{A}^{p}$, and for a given time evolution $\varepsilon(t)$ of the strain tensor, $t \in[0, T]$, we require $\sigma^{p}$ to satisfy the constraint

$$
\sigma^{p}(t) \in Z \quad \forall t \in[0, T]
$$

where $Z \subset \mathbb{R}_{\text {sym }}^{3 \times 3}$ is the domain of admissible plastic stress components. We assume that it has the form

$$
Z=Z_{0} \oplus \operatorname{Lin}\{\mathbf{1}\}
$$

where $\operatorname{Lin}\{\mathbf{1}\}$ is the $1 \mathrm{D}$ space spanned by the Kronecker tensor $\mathbf{1}$ and $Z_{0}$ is a bounded convex closed subset with 0 in its interior of the orthogonal complement $\operatorname{Lin}\{\mathbf{1}\}^{\perp}$ of $\operatorname{Lin}\{\mathbf{1}\}$ (the deviatoric space). The boundary $\partial Z$ of $Z$ is the yield surface. The time evolution of $\varepsilon^{p}$ is governed by the flow rule

$$
\varepsilon_{t}^{p}:\left(\sigma^{p}-\tilde{\sigma}\right) \geq 0 \quad \forall \tilde{\sigma} \in Z
$$

which implies that

$$
\varepsilon_{t}^{p}: \sigma^{p}=M_{Z^{*}}\left(\varepsilon_{t}^{p}\right),
$$

where $M_{Z^{*}}$ is the Minkowski functional of the polar set $Z^{*}$ to $Z$. The physical interpretation of (2.5) is the maximal dissipation principle. Geometrically, it states that the plastic strain rate $\varepsilon_{t}^{p}$ points in the outward normal direction to the yield surface at the point $\sigma^{p}$. Indeed, if $\sigma^{p}$ is in the interior of $Z$, then $\varepsilon_{t}^{p}=0$.

It follows from (2.4) that there exist $\sigma_{0}^{p} \in Z_{0}$ (plastic stress deviator) and $c \in \mathbb{R}$ (pressure) such that $\sigma^{p}=\sigma_{0}^{p}-c \mathbf{1}$. On the other hand, putting in (2.5) $\tilde{\sigma}=\sigma_{0}^{p}-\rho \mathbf{1}$ for an arbitrary $\rho \in \mathbb{R}$, we obtain $\varepsilon_{t}^{p}: \mathbf{1}=0$ (in other words, no volume changes occur during plastic deformation), so that

$$
M_{Z^{*}}\left(\varepsilon_{t}^{p}\right)=\varepsilon_{t}^{p}: \sigma_{0}^{p} \leq \operatorname{diam}\left(Z_{0}\right)\left|\varepsilon_{t}^{p}\right|
$$


We can eliminate the internal variables $\varepsilon^{e}, \varepsilon^{p}$ and write (2.5) in the form

$$
\left(\varepsilon_{t}-\left(\mathbf{A}^{p}\right)^{-1} \sigma_{t}^{p}\right):\left(\sigma^{p}-\tilde{\sigma}\right) \geq 0 \quad \forall \tilde{\sigma} \in Z .
$$

We now define a new scalar product $\langle\cdot, \cdot\rangle_{\mathbf{A}^{p}}$ in $\mathbb{R}_{\mathrm{sym}}^{3 \times 3}$ by the formula $\langle\xi, \eta\rangle_{\mathbf{A}^{p}}=\left(\mathbf{A}^{p}\right)^{-1} \xi: \eta$ for generic tensors $\xi, \eta$, and rewrite (2.8) as

$$
\left\langle\mathbf{A}^{p} \varepsilon_{t}-\sigma_{t}^{p}, \sigma^{p}-\tilde{\sigma}\right\rangle_{\mathbf{A}^{p}} \geq 0 \quad \forall \tilde{\sigma} \in Z .
$$

We prescribe a canonical initial condition for $\sigma^{p}$, namely

$$
\sigma^{p}(0)=\operatorname{Proj}_{Z}\left(\mathbf{A}^{p} \varepsilon(0)\right),
$$

where $\operatorname{Proj}_{Z}$ is the orthogonal projection $\mathbb{R}_{\text {sym }}^{3 \times 3} \rightarrow Z$ with respect to the scalar product $\langle\cdot, \cdot\rangle_{\mathbf{A}^{p}}$ and is characterized by the variational inequality

$$
x=\operatorname{Proj}_{Z}(u) \Longleftrightarrow x \in Z,\langle u-x, x-y\rangle_{\mathbf{A}^{p}} \geq 0 \quad \forall y \in Z .
$$

We list here some properties of the variational problem (2.3), (2.9), (2.10). The proof can be found in [15, Chapter I].

Proposition 2.1. For every $\varepsilon \in W^{1,1}\left(0, T ; \mathbb{R}_{\mathrm{sym}}^{3 \times 3}\right)$ there exists a unique $\sigma^{p} \in W^{1,1}\left(0, T ; \mathbb{R}_{\mathrm{sym}}^{3 \times 3}\right)$ satisfying (2.3), (2.9), (2.10). The solution mapping

$$
P_{0}: W^{1,1}\left(0, T ; \mathbb{R}_{\mathrm{sym}}^{3 \times 3}\right) \rightarrow W^{1,1}\left(0, T ; \mathbb{R}_{\mathrm{sym}}^{3 \times 3}\right): \varepsilon \mapsto \sigma^{p}
$$

has the following properties.

(i) For all $\varepsilon \in W^{1,1}\left(0, T ; \mathbb{R}_{\mathrm{sym}}^{3 \times 3}\right)$ we have $\left|P_{0}[\varepsilon]_{t}\right| \leq\left|\varepsilon_{t}\right|$ a.e., $P_{0}: W^{1,1}\left(0, T ; \mathbb{R}_{\mathrm{sym}}^{3 \times 3}\right) \rightarrow$ $W^{1,1}\left(0, T ; \mathbb{R}_{\mathrm{sym}}^{3 \times 3}\right)$ is strongly continuous, and admits an extension to a strongly continuous mapping $C\left([0, T] ; \mathbb{R}_{\mathrm{sym}}^{3 \times 3}\right) \rightarrow C\left([0, T] ; \mathbb{R}_{\mathrm{sym}}^{3 \times 3}\right)$.

(ii) There exists a constant $C>0$ such that for every $\varepsilon_{1}, \varepsilon_{2} \in W^{1,1}\left(0, T ; \mathbb{R}_{\mathrm{sym}}^{3 \times 3}\right)$ and every $t \in[0, T]$ we have

$$
\left|P_{0}\left[\varepsilon_{1}\right](t)-P_{0}\left[\varepsilon_{2}\right](t)\right| \leq C\left(\left|\varepsilon_{1}(0)-\varepsilon_{2}(0)\right|+\int_{0}^{t}\left|\left(\varepsilon_{1}\right)_{t}(\tau)-\left(\varepsilon_{2}\right)_{t}(\tau)\right| \mathrm{d} \tau\right) .
$$

(iii) For all $\varepsilon \in W^{1,1}\left(0, T ; \mathbb{R}_{\mathrm{sym}}^{3 \times 3}\right)$, the energy balance equation

$$
P_{0}[\varepsilon]: \varepsilon_{t}-\frac{1}{2}\left(\left(\mathbf{A}^{p}\right)^{-1} P_{0}[\varepsilon]: P_{0}[\varepsilon]\right)_{t}=M_{Z^{*}}\left(\left(\varepsilon-\left(\mathbf{A}^{p}\right)^{-1} P_{0}[\varepsilon]\right)_{t}\right)
$$

is satisfied almost everywhere in $(0, T)$, where $M_{Z^{*}}$ is the Minkowski functional of the polar set $Z^{*}$ to $Z$.

It follows from Proposition 2.1 that the operator $P$ in (1.1) can be represented in the form

$$
P[\varepsilon]=\mathbf{A}^{e} \varepsilon+P_{0}[\varepsilon],
$$

and the first energy identity in (1.3) holds with the choice

$$
V_{P}[\varepsilon]=\frac{1}{2} \mathbf{A}^{e} \varepsilon: \varepsilon+\frac{1}{2}\left(\mathbf{A}^{p}\right)^{-1} P_{0}[\varepsilon]: P_{0}[\varepsilon], \quad D_{P}[\varepsilon]=\varepsilon-\left(\mathbf{A}^{p}\right)^{-1} P_{0}[\varepsilon], \quad\|\cdot\|_{*}=M_{Z^{*}}(\cdot) .
$$




\subsection{The operator $G$}

Similarly as in (2.14), the operator $G$ is considered as a sum

$$
G[p]=f(p)+G_{0}[p],
$$

where $f$ is a monotone function satisfying Hypothesis 3.1(iii) below, and $G_{0}$ is a Preisach operator that we briefly describe here.

The construction of the Preisach operator $G_{0}$ is also based on a variational inequality of the type (2.9). More precisely, for a given input function $p \in W^{1,1}(0, T)$ and a memory parameter $r>0$, we define the function $\xi_{r}(t)$ as the solution of the variational inequality

$$
\begin{cases}\left|p(t)-\xi_{r}(t)\right| \leq r & \forall t \in[0, T] \\ \left(\xi_{r}\right)_{t}\left(p(t)-\xi_{r}(t)-z\right) \geq 0 & \text { a. e. } \forall z \in[-r, r]\end{cases}
$$

with a prescribed initial condition $\xi_{r}(0) \in[p(0)-r, p(0)+r]$.

This is indeed a scalar version of (2.9) with $Z$ replaced by the interval $[-r, r], \varepsilon$ replaced by $p$, and $\sigma^{p}$ replaced by $p-\xi_{r}$. Here, we consider the whole continuous family of variational inequalities (2.17) parameterized by $r>0$. We introduce the memory state space

$$
\Lambda=\left\{\lambda \in W^{1, \infty}(0, \infty):\left|\lambda^{\prime}(r)\right| \leq 1 \text { a. e. }\right\}
$$

and its subspace

$$
\Lambda_{K}=\{\lambda \in \Lambda: \lambda(r)=0 \text { for } r \geq K\} .
$$

We fix $K>0$ and an initial state $\lambda_{-1} \in \lambda_{K}$, and choose the initial condition as

$$
\xi_{r}(0)=\max \left\{p(0)-r, \min \left\{\lambda_{-1}(r), p(0)+r\right\}\right\} .
$$

We have indeed for all $r>0$ the natural bound

$$
\left|\xi_{r}(0)\right| \leq \max \{|p(0)|, K\} .
$$

The mapping $\mathfrak{p}_{r}: W^{1,1}(0, T) \rightarrow W^{1,1}(0, T)$ which with each $p \in W^{1,1}(0, T)$ associates the solution $\xi_{r}=\mathfrak{p}_{r}[p] \in W^{1,1}(0, T)$ of (2.17), (2.20) is called the play. This concept goes back to [14], and the proof of the following statements can be found, e. g., in [15, Chapter II].

Proposition 2.2. For each $r>0$, the mapping $\mathfrak{p}_{r}: W^{1,1}(0, T) \rightarrow W^{1,1}(0, T)$ is Lipschitz continuous and admits a Lipschitz continuous extension to $\mathfrak{p}_{r}: C[0, T] \rightarrow C[0, T]$ in the sense that for every $p_{1}, p_{2} \in C[0, T]$ and every $t \in[0, T]$ we have

$$
\left|\mathfrak{p}_{r}\left[p_{1}\right](t)-\mathfrak{p}_{r}\left[p_{2}\right](t)\right| \leq \max _{\tau \in[0, t]}\left|p_{1}(\tau)-p_{2}(\tau)\right|
$$

Moreover, for each $p \in W^{1,1}(0, T)$, the energy balance equation

$$
\mathfrak{p}_{r}[p]_{t} p-\frac{1}{2}\left(\mathfrak{p}_{r}^{2}[p]\right)_{t}=\left|r \mathfrak{p}_{r}[p]_{t}\right|
$$

and the identity

$$
\mathfrak{p}_{r}[p]_{t} p_{t}=\left(\mathfrak{p}_{r}[p]_{t}\right)^{2}
$$

hold almost everywhere in $(0, T)$. 
Proposition 2.3. Let $\lambda_{-1} \in \Lambda_{K}$ be given, and let $\left\{\mathfrak{p}_{r}: r>0\right\}$ be the family of play operators. Then for every $p \in C[0, T]$ and every $t \in[0, T]$ we have

(i) $\mathfrak{p}_{r}[p](t)=0$ for $r \geq K^{*}(t):=\max \left\{K, \max _{\tau \in[0, t]}|p(\tau)|\right\}$;

(ii) The function $r \mapsto \mathfrak{p}_{r}[p](t)$ belongs to $\Lambda_{K^{*}(t)}$.

Given a nonnegative function $\rho \in L^{1}((0, \infty) \times \mathbb{R})$ (the Preisach density), we define the Preisach operator $G_{0}$ as a mapping that with each $p \in C[0, T]$ associates the integral

$$
G_{0}[p](t)=\int_{0}^{\infty} \int_{0}^{\mathfrak{p}_{r}[p](t)} \rho(r, v) \mathrm{d} v \mathrm{~d} r
$$

For our purposes, we adopt the following hypothesis on the Preisach density.

Hypothesis 2.4. There exists a function $\rho^{*} \in L^{1}(0, \infty)$ such that for a. e. $v \in \mathbb{R}$ we have $0 \leq \rho(r, v) \leq \rho^{*}(r)$, and we put

$$
C_{\rho}=\int_{0}^{\infty} \int_{-\infty}^{\infty} \rho(r, v) \mathrm{d} v \mathrm{~d} r, \quad C_{\rho}^{*}=\int_{0}^{\infty} \rho^{*}(r) \mathrm{d} r
$$

For the reader who is more familiar with the original Preisach construction in [20] based on non-ideal relays, let us just point out that for integrable densities, the variational setting in (2.25) is equivalent, as shown in [16].

From (2.23), (2.24), and (2.25) we immediately deduce the Preisach energy identity

$$
G_{0}[p]_{t} p-V_{0}[p]_{t}=\left|D_{0}[p]_{t}\right| \text { a. e. }
$$

provided we define the Preisach potential $V_{0}$ and the dissipation operator $D_{0}$ by the integrals

$$
V_{0}[p](t)=\int_{0}^{\infty} \int_{0}^{\mathfrak{p}_{r}[p](t)} v \rho(r, v) \mathrm{d} v \mathrm{~d} r, \quad D_{0}[p](t)=\int_{0}^{\infty} \int_{0}^{\mathfrak{p}_{r}[p](t)} r \rho(r, v) \mathrm{d} v \mathrm{~d} r .
$$

The second identity in (1.3) then holds with the choice

$$
V_{G}[p]=p f(p)-\int_{0}^{p} f(z) \mathrm{d} z+V_{0}[p], \quad D_{G}[p]=D_{0}[p] .
$$

A straightforward computation shows that $G_{0}$ (and, consequently, $G$ ) are Lipschitz continuous in $C[0, T]$. Indeed, using (2.22) and Hypothesis 2.4, we obtain for $p_{1}, p_{2} \in C[0, T]$ and $t \in[0, T]$ that

$$
\left|G_{0}\left[p_{2}\right](t)-G_{0}\left[p_{1}\right](t)\right|=\left|\int_{0}^{\infty} \int_{\mathfrak{p}_{r}\left[p_{1}\right](t)}^{\mathfrak{p}_{r}\left[p_{2}\right](t)} \rho(v, r) \mathrm{d} v \mathrm{~d} r\right| \leq C_{\rho}^{*} \max _{\tau \in[0, t]}\left|p_{2}(\tau)-p_{1}(\tau)\right| .
$$

We similarly get, using (2.21), bounds for the initial time $t=0$, namely

$$
\begin{aligned}
\left|G_{0}[p](0)\right| & =\left|\int_{0}^{\infty} \int_{0}^{\mathfrak{p}_{r}[p](0)} \rho(v, r) \mathrm{d} v \mathrm{~d} r\right| \leq \min \left\{C_{\rho}, C_{\rho}^{*} \max \{|p(0)|, K\}\right\}, \\
\left|V_{0}[p](0)\right| & =\left|\int_{0}^{\infty} \int_{0}^{\mathfrak{p}_{r}[p](0)} v \rho(v, r) \mathrm{d} v \mathrm{~d} r\right| \leq C_{\rho} \max \{|p(0)|, K\} .
\end{aligned}
$$


The Preisach operator admits also a family of "nonlinear" energies. As a consequence of (2.23), we have for a. e. $t$ the inequality

$$
\mathfrak{p}_{r}[p]_{t}\left(p-\mathfrak{p}_{r}[p]\right) \geq 0
$$

hence

$$
\mathfrak{p}_{r}[p]_{t}\left(h(p)-h\left(\mathfrak{p}_{r}[p]\right)\right) \geq 0
$$

for every nondecreasing function $h: \mathbb{R} \rightarrow \mathbb{R}$. Hence, for every absolutely continuous input $p$, a counterpart of (2.27) in the form

$$
G_{0}[p]_{t} h(p)-V_{h}[p]_{t} \geq 0 \text { a. e. }
$$

holds with a modified potential

$$
V_{h}[p](t)=\int_{0}^{\infty} \int_{0}^{\mathfrak{p}_{r}[p](t)} h(v) \rho(r, v) \mathrm{d} v \mathrm{~d} r
$$

This is related to the fact that for every absolutely continuous nondecreasing function $\hat{h}: \mathbb{R} \rightarrow$ $\mathbb{R}$, the mapping $G_{\hat{h}}:=G_{0} \circ \hat{h}$ is also a Preisach operator, see [17].

\section{Main results}

We denote

$$
X_{0}=\left\{\phi \in W^{1,2}\left(\Omega ; \mathbb{R}^{3}\right):\left.\phi\right|_{\partial \Omega}=0\right\}, \quad X=W^{1,2}(\Omega), \quad X_{q}=W^{1, q}(\Omega)
$$

for $q>1$, and reformulate Problem (1.4)-(1.6) in variational form for all test functions $\phi \in X_{0}$, $\psi \in X$, and $\zeta \in X_{q^{*}}$ for a suitable $q^{*}>2$ as follows:

$$
\begin{aligned}
\int_{\Omega}\left(\rho_{S} u_{t t} \cdot \phi+\left(\mathbf{B} \nabla_{s} u_{t}+P\left[\nabla_{s} u\right]\right): \nabla_{s} \phi+(p-\beta \theta) \operatorname{div} \phi\right) \mathrm{d} x & =\int_{\Omega} g \cdot \phi \mathrm{d} x \\
\int_{\Omega}\left(\left(G[p]_{t}-\operatorname{div} u_{t}\right) \psi+\frac{1}{\rho_{L}} \mu(p) \nabla p \nabla \psi\right) \mathrm{d} x & =\int_{\partial \Omega} \gamma_{p}(x)\left(p^{*}-p\right) \psi \mathrm{d} s(x), \\
\int_{\Omega}\left(\left(c_{0} \theta_{t}-\left\|D_{P}\left[\nabla_{s} u\right]_{t}\right\|_{*}-\left|D_{G}[p]_{t}\right|\right) \zeta+\kappa(\theta) \nabla \theta \cdot \nabla \zeta\right) \mathrm{d} x & \\
-\int_{\Omega}\left(\mathbf{B} \nabla_{s} u_{t}: \nabla_{s} u_{t}+\frac{1}{\rho_{L}} \mu(p)|\nabla p|^{2}-\beta \theta \operatorname{div} u_{t}\right) \zeta \mathrm{d} x & =\int_{\partial \Omega} \gamma_{\theta}(x)\left(\theta^{*}-\theta\right) \zeta \mathrm{d} s(x) .
\end{aligned}
$$

Hypothesis 3.1. We assume that $\Omega$ is a bounded domain with $C^{1,1}$ boundary. We fix an arbitrary final time $T>0$, a constant $\bar{\theta}>0$, and functions $p^{*} \in W^{1, \infty}(\partial \Omega \times(0, T)), \theta^{*} \in$ $L^{\infty}(\partial \Omega \times(0, T))$ such that $\theta^{*}(x, t) \geq \bar{\theta}, g \in L^{2}(\Omega \times(0, T)), \gamma_{\theta} \in L^{\infty}(\partial \Omega), \gamma_{p} \in W^{1, \infty}(\partial \Omega)$, $\gamma_{\theta} \geq 0, \gamma_{p} \geq 0$ a. e., $\int_{0}^{T} \int_{\partial \Omega} \gamma_{p}(x) \mathrm{d} s(x)>0, \beta \in \mathbb{R}, c_{0}>0$. The coefficients $\rho_{S}, \rho_{L}$ are constant and positive, and $\mathbf{B}$ is the isotropic symmetric positive definite fourth order tensor of the form

$$
\mathbf{B}_{i j k l}=2 \eta \delta_{i k} \delta_{j l}+\omega \delta_{k l} \delta_{i j}
$$

with constants $\eta>0, \omega>0$. The nonlinearities in (3.2)-(3.4) satisfy the following conditions 
(i) $\mu: \mathbb{R} \rightarrow\left[\mu_{0}, \mu_{1}\right]$ is a $C^{1}$ function, $0<\mu_{0}<\mu_{1}$ are fixed constants, and we set

$$
M(p)=\int_{0}^{p} \mu\left(p^{\prime}\right) \mathrm{d} p^{\prime}
$$

(ii) $\kappa: \mathbb{R} \rightarrow(0, \infty)$ is a $C^{1}$ function, $\kappa(0)>0$, and there exist constants $0<a<b<\frac{27}{5}+\frac{12}{5} a$ such that

$$
\liminf _{\theta \rightarrow \infty} \frac{\kappa(\theta)}{\theta^{1+a}}>0, \quad \limsup _{\theta \rightarrow \infty} \frac{\kappa(\theta)}{\theta^{1+b}}<\infty .
$$

(iii) $G[p]=f(p)+G_{0}[p]$, where $G_{0}$ is the Preisach operator from Subsection 2.2 with an initial memory state $\lambda_{-1} \in \Lambda_{K}$ for some $K \geq \sup \left|p^{*}\right|$. The dissipation operator $D_{G}$ associated with $G$ is defined in (2.28) (2.29), and $f: \mathbb{R} \rightarrow\left(0, f_{1}\right)$ for some $f_{1}>0$ is a $C^{1}$ function such that there exist $0<f_{2}<f_{3}$ with the property

$$
f_{2} \leq f^{\prime}(p)\left(1+p^{2}\right) \leq f_{3} \quad \forall p \in \mathbb{R} .
$$

(iv) Let $\mathbb{R}_{\mathrm{sym}}^{3 \times 3}$ denote the space of symmetric $3 \times 3$ tensors. We assume that the operator $P: C\left([0, T] ; \mathbb{R}_{\mathrm{sym}}^{3 \times 3}\right) \rightarrow C\left([0, T] ; \mathbb{R}_{\mathrm{sym}}^{3 \times 3}\right)$ has the form $(2.14)$ with $P_{0}$ defined in Proposition 2.1, and with dissipation operator $D_{P}$ defined in (2.15).

We prescribe initial conditions (1.7) with $u^{0} \in X_{0} \cap W^{2,2}\left(\Omega ; \mathbb{R}^{3}\right), u^{1} \in X_{0}, p^{0} \in L^{\infty}(\Omega) \cap$ $W^{1,2}(\Omega),\left|p^{0}(x)\right| \leq K$ a. e., $\theta^{0} \in L^{\infty}(\Omega), \theta^{0}(x) \geq \bar{\theta}$ a. e.

Condition (ii) in Hypothesis 3.1 is a slight generalization of Hypothesis (I) of [21]. We will see the role that it plays in the existence proof.

The main result of this paper reads as follows.

Theorem 3.2. Let Hypotheses 2.4, 3.1 hold. Then there exists $q^{*}$ depending on $a$ and $b$ and $a$ solution $(u, p, \theta)$ to (3.2) -(3.4), and (1.7) with the properties $u_{t} \in L^{2}\left(0, T ; X_{0} \cap W^{2,2}\left(\Omega ; \mathbb{R}^{3}\right)\right)$, $u_{t t} \in L^{2}(\Omega \times(0, T)), p \in L^{\infty}(\Omega \times(0, T)), M(p) \in L^{2}\left(0, T ; W^{2,2}(\Omega)\right)$ with $M(p)$ given by (3.6) $, p_{t} \in L^{2}(\Omega \times(0, T)), \theta \in L^{z}(\Omega \times(0, T))$ for every $z<8+3 a, \nabla \theta \in L^{2}(\Omega \times(0, T))$, $\theta_{t} \in L^{2}\left(0, T ; W^{-1, q^{*}}(\Omega)\right)$ for some $q^{*}>2$.

We first regularize the problem, prove the existence of a solution for the regularized system, derive estimates independent of the regularization parameters, and pass to the limit.

\section{Regularization}

We choose regularizing parameters $R>K$ with $K$ from Hypothesis 2.4 and $\delta>0$ with the intention to let $R \rightarrow \infty$ and $\delta \rightarrow 0$, and define mappings $Q_{R}: \mathbb{R} \rightarrow[0, R]$ and $K_{R}: \mathbb{R} \rightarrow \mathbb{R}$ by the formulas

$$
Q_{R}(z)=\max \{0, \min \{z, R\}\}, \quad K_{R}(z)=\max \{z-R, \min \{0, z+R\}\} \text { for } z \in \mathbb{R} .
$$


Let $\mathcal{B}: W^{2,2}\left(\Omega ; \mathbb{R}^{3}\right) \cap X_{0} \rightarrow L^{2}\left(\Omega ; \mathbb{R}^{3}\right)$ denote the mapping

$$
\mathcal{B} v=-\operatorname{div} \mathbf{B} \nabla_{s} v
$$

It follows from a vector counterpart of [12, Lemma 9.17], cf. also the methods proposed in [19, Lemma 3.2, p. 260], that $|\mathcal{B} v|_{2}$ is an equivalent norm for $v$ in $W^{2,2}(\Omega) \cap X_{0}$, that is, there exist positive constants $C_{1}<C_{2}$ such that for every $v \in W^{2,2}\left(\Omega ; \mathbb{R}^{3}\right) \cap X_{0}$ we have

$$
C_{1}\|v\|_{W^{2,2}(\Omega)} \leq|\mathcal{B} v|_{2} \leq C_{2}\|v\|_{W^{2,2}(\Omega)} .
$$

We replace (3.2)- (3.4) by the system

$$
\begin{aligned}
\int_{\Omega}\left(\rho_{S} u_{t t} \cdot \phi+\delta \mathcal{B} u_{t t} \cdot \mathcal{B} \phi+\left(\mathbf{B} \nabla_{s} u_{t}+P\left[\nabla_{s} u\right]\right): \nabla_{s} \phi\right) \mathrm{d} x & \\
+\int_{\Omega}\left(p-\beta Q_{R}(\theta)\right) \operatorname{div} \phi \mathrm{d} x & =\int_{\Omega} g \cdot \phi \mathrm{d} x \\
\int_{\Omega}\left(\left(\left(K_{R}(p)+G[p]\right)_{t}-\operatorname{div} u_{t}\right) \psi+\frac{1}{\rho_{L}} \mu(p) \nabla p \nabla \psi\right) \mathrm{d} x & =\int_{\partial \Omega} \gamma_{p}(x)\left(p^{*}-p\right) \psi \mathrm{d} s(x), \\
\int_{\Omega}\left(\left(c_{0} \theta_{t}-\left\|D_{P}\left[\nabla_{s} u\right]_{t}\right\|_{*}-\left|D_{G}[p]_{t}\right|\right) \zeta+\kappa(\theta) \nabla \theta \cdot \nabla \zeta\right) \mathrm{d} x & \\
-\int_{\Omega}\left(\mathbf{B} \nabla_{s} u_{t}: \nabla_{s} u_{t}+\frac{1}{\rho_{L}} \mu(p) Q_{R}\left(|\nabla p|^{2}\right)-\beta Q_{R}(\theta) \operatorname{div} u_{t}\right) \zeta \mathrm{d} x & =\int_{\partial \Omega} \gamma_{\theta}(x)\left(\theta^{*}-\theta\right) \zeta \mathrm{d} s(x)
\end{aligned}
$$

with test functions $\phi \in W^{2,2}\left(\Omega ; \mathbb{R}^{3}\right) \cap X_{0}, \psi, \zeta \in X$ and initial conditions (1.7).

Proposition 4.1. In addition to Hypotheses 2.4, 3.1, assume that $u^{1} \in X_{0} \cap W^{2,2}\left(\Omega ; \mathbb{R}^{3}\right)$. Then there exists a solution $(u, p, \theta)$ to (4.4) -(4.6) with the properties $u_{t t} \in L^{2}\left(0, T ; W^{2,2}\left(\Omega ; \mathbb{R}^{3}\right) \cap\right.$ $\left.X_{0}\right), p \in L^{2}\left(0, T ; W^{1,2}(\Omega)\right), M(p) \in L^{2}\left(0, T ; W^{2,2}(\Omega)\right)$ with $M(p)$ given by (3.6) $), p_{t} \in L^{2}(\Omega \times$ $(0, T)), \theta \in L^{2}(\Omega \times(0, T)), \nabla \theta \in L^{2}(\Omega \times(0, T)), \theta_{t} \in L^{2}\left(0, T ; X^{*}\right)$, where $X^{*}$ is the dual of $X$.

System (4.4)-(4.6) for each fixed $R>0$ and $\delta>0$ will be solved by Faedo-Galerkin approximations. We choose $\mathcal{E}=\left\{e_{k} ; k=1,2, \ldots\right\}$ in $L^{2}\left(\Omega ; \mathbb{R}^{3}\right)$ and $\mathcal{W}=\left\{w_{j} ; j=0,1,2, \ldots\right\}$ in $L^{2}(\Omega)$ to be the complete orthonormal systems of eigenfunctions defined by

$$
\mathcal{B} e_{k}=\lambda_{k} e_{k} \text { in } \Omega,\left.\quad e_{k}\right|_{\partial \Omega}=0, \quad-\Delta w_{j}=\mu_{j} w_{j} \text { in } \Omega,\left.\quad \nabla w_{j} \cdot n\right|_{\partial \Omega}=0,
$$

with $\mu_{0}=0, \lambda_{k}>0, \mu_{j}>0$ for $j, k \geq 1$, and put for $n \in \mathbb{N}$

$$
u^{(n)}(x, t)=\sum_{k=1}^{n} u_{k}(t) e_{k}(x), \quad \theta^{(n)}(x, t)=\sum_{j=0}^{n} \theta_{j}(t) w_{j}(x)
$$

with coefficients $u_{k}:[0, T] \rightarrow \mathbb{R}, \theta_{j}:[0, T] \rightarrow \mathbb{R}$ which will be determined as the solution of 
the system

$$
\begin{aligned}
\left(\rho_{S}+\lambda_{k}+\delta \lambda_{k}^{2}\right) \ddot{u}_{k}+\int_{\Omega} P\left[\nabla_{s} u^{(n)}\right]: \nabla_{s} e_{k} \mathrm{~d} x & \\
+\int_{\Omega}\left(p^{(n)}-\beta Q_{R}\left(\theta^{(n)}\right)\right) \operatorname{div} e_{k} \mathrm{~d} x & =\int_{\Omega} g \cdot e_{k} \mathrm{~d} x \\
\int_{\Omega}\left(\left(K_{R}\left(p^{(n)}\right)+G\left[p^{(n)}\right]\right)_{t}-\operatorname{div} u_{t}^{(n)}\right) \psi \mathrm{d} x & \\
+\frac{1}{\rho_{L}} \int_{\Omega} \mu\left(p^{(n)}\right) \nabla p^{(n)} \cdot \nabla \psi \mathrm{d} x & =\int_{\partial \Omega} \gamma_{p}(x)\left(p^{*}-p^{(n)}\right) \psi \mathrm{d} s(x), \\
c_{0} \dot{\theta}_{j}+\int_{\Omega}\left(-\left|D_{G}\left[p^{(n)}\right]_{t}\right| w_{j}+\kappa\left(\theta^{(n)}\right) \nabla \theta^{(n)} \cdot \nabla w_{j}\right) \mathrm{d} x & \\
+\int_{\Omega}\left(\beta Q_{R}\left(\theta^{(n)}\right) \operatorname{div} u_{t}^{(n)}-\left\|D_{P}\left[\nabla_{s} u^{(n)}\right] t\right\|_{*}\right) w_{j} \mathrm{~d} x & \\
-\int_{\Omega}\left(\mathbf{B} \nabla_{s} u_{t}^{(n)}: \nabla_{s} u_{t}^{(n)}+\frac{1}{\rho_{L}} \mu\left(p^{(n)}\right) Q_{R}\left(\left|\nabla p^{(n)}\right|^{2}\right)\right) w_{j} \mathrm{~d} x & =\int_{\partial \Omega} \gamma_{\theta}(x)\left(\theta^{*}-\theta^{(n)}\right) w_{j} \mathrm{~d} s(x)
\end{aligned}
$$

for $k=1, \ldots, n$ and $j=0,1, \ldots, n$, and for all $\psi \in X$. We prescribe initial conditions

$$
\begin{aligned}
u_{k}(0)=\int_{\Omega} u^{0}(x) \cdot e_{k}(x) \mathrm{d} x, & \dot{u}_{k}(0)=\int_{\Omega} u^{1}(x) \cdot e_{k}(x) \mathrm{d} x, \\
\theta_{j}(0)=\int_{\Omega} \theta^{0}(x) w_{j}(x) \mathrm{d} x, & p^{(n)}(x, 0)=p^{0}(x) .
\end{aligned}
$$

This is an ODE system (4.9), (4.11) coupled with a standard PDE with hysteresis (4.10), which has a strong solution in a maximal interval of existence, which coincides with the whole interval $[0, T]$ provided we prove that the solution remains bounded in the maximal interval of existence.

Put $\mathcal{E}_{n}=\left\{e_{k} ; k=1,2, \ldots, n\right\}$ and $\mathcal{W}_{n}=\left\{w_{j} ; j=0,1,2, \ldots, n\right\}$. Then (4.9)-(4.11) can be equivalently written as

$$
\begin{aligned}
\int_{\Omega}\left(\rho_{S} u_{t t}^{(n)} \cdot \phi+\left(\mathbf{B} \nabla_{s} u_{t}^{(n)}+P\left[\nabla_{s} u^{(n)}\right]\right): \nabla_{s} \phi\right) \mathrm{d} x & \\
+\delta \int_{\Omega} \mathcal{B} u_{t t}^{(n)} \cdot \mathcal{B} \phi \mathrm{d} x+\int_{\Omega}\left(p^{(n)}-\beta Q_{R}\left(\theta^{(n)}\right)\right) \operatorname{div} \phi \mathrm{d} x & =\int_{\Omega} g \cdot \phi \mathrm{d} x \\
\int_{\Omega}\left(\left(K_{R}\left(p^{(n)}\right)+G\left[p^{(n)}\right]\right)_{t}-\operatorname{div} u_{t}^{(n)}\right) \psi \mathrm{d} x & \\
+\int_{\Omega} \frac{1}{\rho_{L}} \mu\left(p^{(n)}\right) \nabla p^{(n)} \nabla \psi \mathrm{d} x & =\int_{\partial \Omega} \gamma_{p}(x)\left(p^{*}-p^{(n)}\right) \psi \mathrm{d} s(x), \\
\int_{\Omega}\left(\left(c_{0} \theta_{t}^{(n)}-\left|D_{G}\left[p^{(n)}\right]_{t}\right|\right) \zeta+\kappa\left(\theta^{(n)}\right) \nabla \theta^{(n)} \cdot \nabla \zeta\right) \mathrm{d} x & \\
+\int_{\Omega}\left(\beta Q_{R}\left(\theta^{(n)}\right) \operatorname{div} u_{t}^{(n)}-\left\|D_{P}\left[\nabla_{s} u^{(n)}\right] t\right\|_{*}\right) \zeta \mathrm{d} x & \\
-\int_{\Omega}\left(\mathbf{B} \nabla_{s} u_{t}^{(n)}: \nabla_{s} u_{t}^{(n)}+\frac{1}{\rho_{L}} \mu\left(p^{(n)}\right) Q_{R}\left(\left|\nabla p^{(n)}\right|^{2}\right)\right) \zeta \mathrm{d} x & =\int_{\partial \Omega} \gamma_{\theta}(x)\left(\theta^{*}-\theta^{(n)}\right) \zeta \mathrm{d} s(x)
\end{aligned}
$$

with test functions $\phi \in \operatorname{Span} \mathcal{E}_{n}, \zeta \in \operatorname{Span} \mathcal{W}_{n}$, and $\psi \in X$. 
We now derive a series of estimates. By $C$ we denote any positive constant depending only on the data, by $C_{R}$ any constant depending on the data and on $R$, and by $C_{R, \delta}$ any constant depending on the data, on $R$, and on $\delta$, all independent of the dimension $n$ of the Galerkin approximation.

To simplify the presentation, we introduce from now on the notation $|\cdot|_{q}$ for the norm in $L^{q}(\Omega)$, and by $\|\cdot\|_{q}$ the norm in $L^{q}(\Omega \times(0, T))$. We will systematically use the Gagliardo-Nirenberg inequality in the form

$$
|w|_{q} \leq C\left(|w|_{s}+|w|_{s}^{1-\gamma}|\nabla w|_{r}^{\gamma}\right), \quad \gamma=\frac{\frac{1}{s}-\frac{1}{q}}{\frac{1}{3}+\frac{1}{s}-\frac{1}{r}}
$$

which holds for every $w \in W^{1, r}(\Omega)$ and every $\frac{1}{s}>\frac{1}{q}>\frac{1}{r}-\frac{1}{3}$. For the proof, see, e.g., [8, $\left.\S 15\right]$.

\section{$4.1 \quad$ Estimate 1}

We test (4.14) with $\phi=u_{t}^{(n)}$ and (4.15) with $\psi=p^{(n)}$ and sum up the results to obtain

$$
\begin{aligned}
\int_{\Omega}\left(\rho_{S} u_{t t}^{(n)} \cdot u_{t}^{(n)}+\delta \mathcal{B} u_{t t}^{(n)} \cdot \mathcal{B} u_{t}^{(n)}+\left(\mathbf{B} \nabla_{s} u_{t}^{(n)}+P\left[\nabla_{s} u^{(n)}\right]\right): \nabla_{s} u_{t}^{(n)}\right. \\
\left.+\left(K_{R}\left(p^{(n)}\right)+G\left[p^{(n)}\right]\right)_{t} p^{(n)}+\frac{1}{\rho_{L}} \mu\left(p^{(n)}\right)\left|\nabla p^{(n)}\right|^{2}\right) \mathrm{d} x \\
=\int_{\Omega}\left(\beta Q_{R}\left(\theta^{(n)}\right) \operatorname{div} u_{t}^{(n)}+g \cdot u_{t}^{(n)}\right) \mathrm{d} x+\int_{\partial \Omega} \gamma_{p}(x) p^{(n)}\left(p^{*}-p^{(n)}\right) \mathrm{d} s(x) .
\end{aligned}
$$

Integrating in time from 0 to $t$ and using the energy identities (1.3) we obtain for all $t \in(0, T)$ the estimate

$$
\begin{aligned}
\left|u_{t}^{(n)}(t)\right|_{2}^{2} & +\delta\left|\mathcal{B} u_{t}^{(n)}(t)\right|_{2}^{2}+\left|\nabla_{s} u^{(n)}(t)\right|_{2}^{2}+\left\|\nabla_{s} u_{t}^{(n)}\right\|_{2}^{2} \\
& +\left|p^{(n)}(t)\right|_{2}^{2}+\left\|\nabla p^{(n)}\right\|_{2}^{2}+\int_{0}^{T} \int_{\partial \Omega} \gamma_{p}(x)\left|p^{(n)}\right|^{2} \mathrm{~d} s(x) \mathrm{d} t \leq C_{R} .
\end{aligned}
$$

\subsection{Estimate 2}

We choose in (4.15) $\psi=M\left(p^{(n)}\right)_{t}$ with $M$ given by (3.6). By Proposition 2.2 and formula (2.25), we have $G_{0}\left[p^{(n)}\right]_{t} M\left(p^{(n)}\right)_{t}=G_{0}\left[p^{(n)}\right]_{t} p_{t}^{(n)} \mu\left(p^{(n)}\right) \geq 0$. By Hypothesis 3.1)(i), (iii), we thus have

$$
\left(K_{R}\left(p^{(n)}\right)+G\left[p^{(n)}\right]_{t}\right) M\left(p^{(n)}\right)_{t} \geq \mu_{0} \min \left\{1, \frac{f_{2}}{1+R^{2}}\right\}\left|p_{t}^{(n)}\right|^{2}
$$

and we obtain for all $t \in(0, T)$ the estimate

$$
\left\|p_{t}^{(n)}\right\|_{2}^{2}+\left|\nabla p^{(n)}(t)\right|_{2}^{2}+\int_{\partial \Omega} \gamma_{p}(x)\left|p^{(n)}(t)\right|^{2} \mathrm{~d} s(x) \leq C_{R}
$$

By comparison in Eq. (4.15), we see that

$$
\left\|M\left(p^{(n)}\right)\right\|_{L^{2}\left(0, T ; W^{2,2}(\Omega)\right)} \leq C_{R} .
$$




\subsection{Estimate 3}

Choosing in (4.14) $\phi=u_{t t}^{(n)}$ yields

$$
\begin{gathered}
\int_{\Omega}\left(\rho_{S}\left|u_{t t}^{(n)}\right|^{2}+\delta\left|\mathcal{B} u_{t t}^{(n)}\right|^{2}+\mathbf{B} \nabla_{s} u_{t}^{(n)}: \nabla_{s} u_{t t}^{(n)}+\frac{\partial}{\partial t}\left(P\left[\nabla_{s} u^{(n)}\right]: \nabla_{s} u_{t}^{(n)}\right)\right) \mathrm{d} x \\
=\int_{\Omega}\left(P\left[\nabla_{s} u^{(n)}\right]_{t}: \nabla_{s} u_{t}^{(n)}+\left(\beta Q_{R}\left(\theta^{(n)}\right)-p^{(n)}\right) \operatorname{div} u_{t t}^{(n)}+g \cdot u_{t t}^{(n)}\right) \mathrm{d} x .
\end{gathered}
$$

We now integrate in time again and use Proposition 2.1, estimate (4.20), as well as the Gronwall argument, to conclude for all $t \in(0, T)$ that

$$
\left\|u_{t t}^{(n)}\right\|_{2}^{2}+\delta\left\|\mathcal{B} u_{t t}^{(n)}\right\|_{2}^{2}+\left|\nabla_{s} u_{t}^{(n)}(t)\right|_{2}^{2} \leq C_{R, \delta}
$$

\subsection{Estimate 4}

We choose in (4.16) $\zeta=\theta^{(n)}$. The only superlinear term in (4.16) is $\mathbf{B} \nabla_{s} u_{t}^{(n)}: \nabla_{s} u_{t}^{(n)}$ which has to be estimated in the norm of $L^{2}(\Omega \times(0, T))$, that is, $\nabla_{s} u_{t}^{(n)}$ has to be estimated in $L^{4}(\Omega \times(0, T))$. This will be done using the Gagliardo-Nirenberg inequality (4.17), which yields for every $t \in(0, T)$ that

$$
\left|\nabla_{s} u_{t}^{(n)}(t)\right|_{4} \leq C\left(\left|\nabla_{s} u_{t}^{(n)}(t)\right|_{2}+\left|\nabla_{s} u_{t}^{(n)}(t)\right|_{2}^{1 / 4}\left|\mathcal{B} u_{t}^{(n)}(t)\right|_{2}^{3 / 4}\right) \leq C_{R, \delta}
$$

by virtue of (4.19) and (4.3). Note also that we have the pointwise inequalities

$$
\left|D_{G}\left[p^{(n)}\right]_{t}\right| \leq C\left|p_{t}^{(n)}\right|, \quad\left\|D_{P}\left[\nabla_{s} u^{(n)}\right]_{t}\right\|_{*} \leq C\left|\nabla_{s} u_{t}^{(n)}\right|
$$

which follow from (2.28), (2.24), (2.15), (2.7), and Proposition 2.1)(i). We thus obtain

$$
\left|\theta^{(n)}(t)\right|_{2}^{2}+\left\|\nabla \theta^{(n)}\right\|_{2}^{2}+\int_{0}^{T} \int_{\partial \Omega} \gamma_{\theta}(x)\left|\theta^{(n)}\right|^{2} \mathrm{~d} s(x) \mathrm{d} t \leq C_{R, \delta}
$$

for all $t \in(0, T)$. Finally, let $\zeta \in L^{2}(0, T ; X)$ be arbitrary, $\zeta(x, t)=\sum_{j=0}^{\infty} \zeta_{j}(t) w_{j}(x)$. We test (4.16) with $\zeta=\zeta_{j}(t)$ and obtain using the previous estimates that

$$
\int_{0}^{T} \int_{\Omega} \theta_{t}^{(n)} \zeta \mathrm{d} x \mathrm{~d} t \leq C_{R, \delta}\|\zeta\|_{L^{2}(0, T ; X)}
$$

or, in other words,

$$
\left\|\theta_{t}^{(n)}\right\|_{L^{2}\left(0, T ; X^{*}\right)} \leq C_{R, \delta}
$$

\subsection{Passage to the limit as $n \rightarrow \infty$}

We keep for the moment the regularization parameters $R$ and $\delta$ fixed, and let $n$ tend to $\infty$. By a standard argument based on compact anisotropic embeddings, see [8], we infer, 
passing to a subsequence, if necessary, that there exist functions $(u, p, \theta)$ such that the following convergences take place:

$$
\begin{aligned}
u_{t t}^{(n)} & \rightarrow u_{t t} & \text { weakly in } & L^{2}\left(0, T ; W^{2,2}\left(\Omega ; \mathbb{R}^{3}\right) \cap X_{0}\right), \\
\nabla_{s} u_{t}^{(n)} & \rightarrow \nabla_{s} u_{t} & \text { strongly in } & L^{4}\left(\Omega ; C\left([0, T] ; \mathbb{R}_{\mathrm{sym}}^{3 \times 3}\right)\right) \\
\nabla_{s} u^{(n)} & \rightarrow \nabla_{s} u & \text { strongly in } & L^{4}\left(\Omega ; C\left([0, T] ; \mathbb{R}_{\mathrm{sym}}^{3 \times 3}\right)\right) \\
P\left[\nabla_{s} u^{(n)}\right] & \rightarrow P\left[\nabla_{s} u\right] & \text { strongly in } & L^{4}\left(\Omega ; C\left([0, T] ; \mathbb{R}_{\mathrm{sym}}^{3 \times 3}\right)\right) \\
\left\|D_{P}\left[\nabla_{s} u^{(n)}\right]_{t}\right\|_{*} & \rightarrow\left\|D_{P}\left[\nabla_{s} u\right]_{t}\right\|_{*} & \text { strongly in } & L^{2}(\Omega ; C[0, T]) \\
p^{(n)} & \rightarrow p & \text { strongly in } & L^{4}(\Omega ; C[0, T]) \\
p_{t}^{(n)} & \rightarrow p_{t} & \text { weakly in } & L^{2}(\Omega \times(0, T)) \\
K_{R}\left(p^{(n)}\right)_{t} & \rightarrow K_{R}(p)_{t} & \text { weakly in } & L^{2}(\Omega \times(0, T)) \\
G\left[p^{(n)}\right]_{t} & \rightarrow G[p]_{t} & \text { weakly in } & L^{2}(\Omega \times(0, T)) \\
\left|D_{G}\left[p^{(n)}\right]_{t}\right| & \rightarrow\left|D_{G}[p]_{t}\right| & \text { weakly in } & L^{2}(\Omega \times(0, T)) \\
\nabla p^{(n)} & \rightarrow \nabla_{p} & \text { strongly in } & L^{2}\left(\Omega \times(0, T) ; \mathbb{R}^{3}\right) \\
Q_{R}\left(\left|\nabla p^{(n)}\right|^{2}\right) & \rightarrow Q_{R}\left(|\nabla p|^{2}\right) & \text { strongly in } & L^{2}(\Omega \times(0, T)) \\
\gamma_{p} p^{(n)} & \rightarrow \gamma_{p} p & \text { strongly in } & L^{2}(\partial \Omega \times[0, T]) \\
\theta^{(n)} & \rightarrow \theta & \text { strongly in } & L^{2}(\Omega \times(0, T)) \\
\theta_{t}^{(n)} & \rightarrow \theta_{t} & \text { weakly in } & L^{2}\left(0, T ; X^{*}\right) \\
\nabla \theta^{(n)} & \rightarrow \nabla \theta & \text { weakly in } & L^{2}\left(\Omega \times(0, T) ; \mathbb{R}^{3}\right) \\
\gamma_{\theta} \theta^{(n)} & \rightarrow \gamma_{\theta} \theta & \text { strongly in } & L^{2}(\partial \Omega \times[0, T])
\end{aligned}
$$

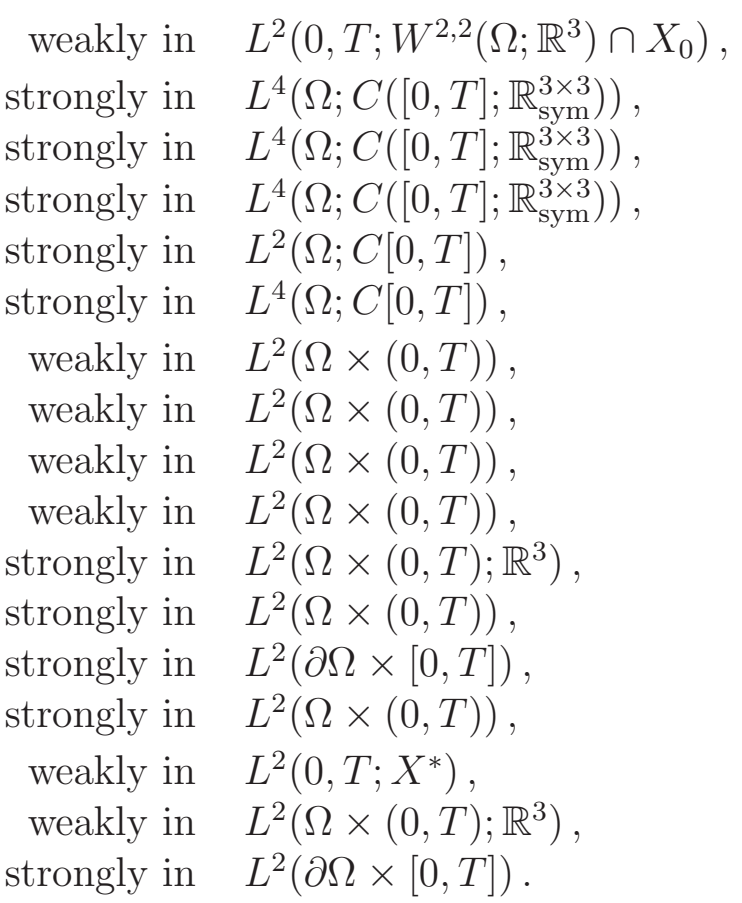

The convergences of the hysteresis terms $P\left[\nabla_{s} u^{(n)}\right], G\left[p^{(n)}\right]_{t},\left\|D_{P}\left[\nabla_{s} u^{(n)}\right]_{t}\right\|_{*},\left|D_{G}\left[p^{(n)}\right]_{t}\right|$ follow indeed from (2.14), (2.12), (2.16), (2.30), and (1.3). We can therefore let $n$ tend to $\infty$ in (4.4)(4.6) and conclude that the limit $(u, p, \theta)$ satisfies the conditions of Proposition 4.1.

\section{Proof of Theorem 3.2}

In this section, we show that a sequence of solutions to (4.4)-(4.6) converges to a solution to (3.2)-(3.4) as $R \rightarrow \infty$ and $\delta \rightarrow 0$. To this end, we fix sequences $\left\{R_{i}\right\},\left\{\delta_{i}\right\}$ for $i \in \mathbb{N}$ such that

$$
\lim _{i \rightarrow \infty} R_{i}=\infty, \quad \lim _{i \rightarrow \infty} \delta_{i}=0,
$$

and choose a sequence $\left\{u_{i}^{1}\right\}$ of initial conditions in $X_{0} \cap W^{3,2}\left(\Omega ; \mathbb{R}^{3}\right)$ such that

$$
\lim _{i \rightarrow \infty}\left\|u_{i}^{1}-u^{1}\right\|_{X_{0}}=0, \quad \lim _{i \rightarrow \infty} \delta_{i}\left\|u_{i}^{1}\right\|_{W^{3,2}\left(\Omega ; \mathbb{R}^{3}\right)}^{2}=0 .
$$

We further denote by $\left(u^{(i)}, p^{(i)}, \theta^{(i)}\right)$ solutions $(u, p, \theta)$ to Problem (4.4)-(4.6) corresponding to the choice $R=R_{i}, \delta=\delta_{i}, u^{1}=u_{i}^{1}$. The next step is to derive some properties of the sequence $\left(u^{(i)}, p^{(i)}, \theta^{(i)}\right)$ independent of $i$. 


\subsection{Positivity of temperature}

We first observe that there exists a constant $C>0$ such that for every nonnegative test function $\zeta \in X$ we have by virtue of (4.6) that

$$
\int_{\Omega}\left(c_{0} \theta_{t}^{(i)} \zeta+\kappa(\theta) \nabla \theta^{(i)} \cdot \nabla \zeta\right) \mathrm{d} x \geq-C \int_{\Omega} Q_{R_{i}}\left(\theta^{(i)}\right)^{2} \zeta \mathrm{d} x+\int_{\partial \Omega} \gamma_{\theta}(x)\left(\theta^{*}-\theta^{(i)}\right) \zeta \mathrm{d} s(x) .
$$

Let $v(t)$ be the solution of the ODE

$$
c_{0} \dot{v}(t)=-C v^{2}(t), \quad v(0)=\bar{\theta},
$$

that is,

$$
v(t)=\left(\frac{C}{c_{0}} t+\frac{1}{\bar{\theta}}\right)^{-1}
$$

For every nonnegative test function $\zeta \in X$ we have in particular

$$
\int_{\Omega}\left(c_{0} v_{t} \zeta+\kappa\left(\theta^{(i)}\right) \nabla v \cdot \nabla \zeta\right) \mathrm{d} x \leq-C \int_{\Omega} v^{2} \zeta \mathrm{d} x+\int_{\partial \Omega} \gamma_{\theta}(x)\left(\theta^{*}-v\right) \zeta \mathrm{d} s(x) .
$$

Subtracting (5.3) from (5.6) we obtain

$$
\int_{\Omega}\left(c_{0}\left(v-\theta^{(i)}\right)_{t} \zeta+\kappa\left(\theta^{(i)}\right) \nabla\left(v-\theta^{(i)}\right) \cdot \nabla \zeta\right) \mathrm{d} x \leq C \int_{\Omega}\left(Q_{R_{i}}^{2}\left(\theta^{(i)}\right)-v^{2}\right) \zeta \mathrm{d} x+\int_{\partial \Omega} \gamma_{\theta}(x)\left(\theta^{(i)}-v\right) \zeta \mathrm{d} s(x) .
$$

We now choose any smooth convex function $F: \mathbb{R} \rightarrow \mathbb{R}$ such that $F(s)=0$ for $s \leq 0$, $F(s)>0$ for $s>0$, and test (5.7) by $\zeta=F^{\prime}\left(v-\theta^{(i)}\right)$. We have in all cases

$$
\left(Q_{R_{i}}^{2}\left(\theta^{(i)}\right)-v^{2}\right) F^{\prime}\left(v-\theta^{(i)}\right) \leq 0 \text { a.e. }
$$

hence

$$
c_{0} \frac{\mathrm{d}}{\mathrm{d} t} \int_{\Omega} F\left(v-\theta^{(i)}\right) \mathrm{d} x \leq 0,
$$

and we conclude for every $i \in \mathbb{N}$ that

$$
\theta^{(i)}(x, t) \geq v(t) \text { a.e. }
$$

We now pass to a series of estimates independent of $i$. To simplify the presentation, we occasionally omit the indices \{\}$^{(i)}$ in the computations in Subsections 5.25 .9 below, and write simply $(u, p, \theta)$ instead of $\left(u^{(i)}, p^{(i)}, \theta^{(i)}\right)$ whenever there is no risk of confusion. The symbol $C$ denotes as before any constant independent of $i$. 


\section{$5.2 \quad$ Estimate 5}

Test (4.4) by $\phi=u_{t}=u_{t}^{(i)}$, (4.5) by $\psi=p=p^{(i)}$, and (4.6) by $\zeta=1$. Summing up the three resulting equations we obtain by virtue of (1.3) that

$$
\begin{aligned}
\frac{\mathrm{d}}{\mathrm{d} t} \int_{\Omega} & \left(c_{0} \theta+\frac{\rho_{S}}{2}\left|u_{t}\right|^{2}+\frac{\delta_{i}}{2}\left|\mathcal{B} u_{t}\right|^{2}+V_{P}\left[\nabla_{s} u\right]+V_{G}[p]+\hat{K}_{R_{i}}(p)\right) \mathrm{d} x \\
& +\frac{1}{\rho_{L}} \int_{\Omega} \mu(p)\left(|\nabla p|^{2}-Q_{R_{i}}\left(|\nabla p|^{2}\right)\right) \mathrm{d} x \\
= & \int_{\Omega} g \cdot u_{t} \mathrm{~d} x+\int_{\partial \Omega}\left(\gamma_{p}(x)\left(p^{*}-p\right) p+\gamma_{\theta}(x)\left(\theta^{*}-\theta\right)\right) \mathrm{d} s(x),
\end{aligned}
$$

where we set $\hat{K}_{R}(p)=\int_{0}^{p} K_{R}^{\prime}\left(p^{\prime}\right) p^{\prime} \mathrm{d} p^{\prime}$ for $p \in \mathbb{R}$ and $R_{i}>0$. Integrating in time and using (2.15) and (5.2), we get for every $t \in(0, T)$ the estimate

$$
\int_{\Omega}\left(\theta^{(i)}+\left|u_{t}^{(i)}\right|^{2}+\left|\nabla_{s} u^{(i)}\right|^{2}\right)(x, t) \mathrm{d} x+\int_{0}^{T} \int_{\partial \Omega}\left(\gamma_{\theta}(x) \theta^{(i)}+\gamma_{p}(x)\left|p^{(i)}\right|^{2}\right)(x, t) \mathrm{d} s(x) \mathrm{d} t \leq C .
$$

\subsection{Estimate 6}

We test (4.6) by $\zeta=\theta^{-a}=\left(\theta^{(i)}\right)^{-a}$ with $a$ from Hypothesis 3.1(ii), and observe that

$$
\begin{aligned}
\int_{\Omega} \theta^{-a} & \left(\left\|D_{P}\left[\nabla_{s} u\right]_{t}\right\|_{*}+\left|D_{G}[p]_{t}\right|+\mathbf{B} \nabla_{s} u_{t}: \nabla_{s} u_{t}+\frac{1}{\rho_{L}} \mu(p) Q_{R_{i}}\left(|\nabla p|^{2}\right)\right) \mathrm{d} x \\
& +a \int_{\Omega} \kappa(\theta) \theta^{-1-a}|\nabla \theta|^{2} \mathrm{~d} x \\
= & \beta \int_{\Omega} Q_{R_{i}}(\theta) \theta^{-a} \operatorname{div} u_{t} \mathrm{~d} x-\int_{\partial \Omega} \gamma_{\theta}(x) \theta^{-a}\left(\theta^{*}-\theta\right) \mathrm{d} s(x)+\frac{c_{0}}{1-a} \frac{\mathrm{d}}{\mathrm{d} t} \int_{\Omega} \theta^{1-a} \mathrm{~d} x
\end{aligned}
$$

Integrating in time and using (5.9), (5.11), and Hypothesis 3.1(ii), we obtain in particular

$$
\int_{0}^{T} \int_{\Omega}\left(\theta^{-a}\left|\operatorname{div} u_{t}\right|^{2}+|\nabla \theta|^{2}\right) \mathrm{d} x \mathrm{~d} t \leq C\left(1+\int_{0}^{T} \int_{\Omega} \theta^{1-a}\left|\operatorname{div} u_{t}\right| \mathrm{d} x \mathrm{~d} t\right) .
$$

The integral on the right hand side can be estimated by Hölder's inequality

$$
\int_{0}^{T} \int_{\Omega} \theta^{1-a}\left|\operatorname{div} u_{t}\right| \mathrm{d} x \mathrm{~d} t \leq\left(\int_{0}^{T} \int_{\Omega} \theta^{-a}\left|\operatorname{div} u_{t}\right|^{2} \mathrm{~d} x \mathrm{~d} t\right)^{1 / 2}\left(\int_{0}^{T} \int_{\Omega} \theta^{2-a} \mathrm{~d} x \mathrm{~d} t\right)^{1 / 2}
$$

which entails that

$$
\int_{0}^{T} \int_{\Omega}|\nabla \theta|^{2} \mathrm{~d} x \mathrm{~d} t \leq C\left(1+\int_{0}^{T} \int_{\Omega} \theta^{2-a} \mathrm{~d} x \mathrm{~d} t\right) .
$$

Applying the Gagliardo-Nirenberg inequality (4.17) with $s=1, r=2$, and $q=2-a$ and using (5.11), we estimate the right hand side of (5.14) from above by $C\left(1+\|\nabla \theta\|_{2}^{(1-a) 6 / 5}\right)$. Thus, for 
$H:=\|\nabla \theta\|_{2}^{2}$, inequality (5.14) has the form $H \leq C\left(H^{\omega}+1\right)$ with $\omega=(1-a) 3 / 5<1$, and $H^{\omega} \leq \omega \delta H+(1-\omega) \delta^{-\omega /(1-\omega)}$. Choosing, for example, $\delta=1 / C$, we obtain

$$
\left\|\nabla \theta^{(i)}\right\|_{2} \leq C .
$$

Using (4.17) again with $s=1, r=2$, and $q=8 / 3$, we obtain

$$
\left\|\theta^{(i)}\right\|_{8 / 3} \leq C .
$$

\subsection{Estimate 7}

Test (4.4) by $\phi=u_{t}=u_{t}^{(i)}$ and (4.5) by $\psi=p=p^{(i)}$. The sum of the two equations yields

$$
\begin{aligned}
& \frac{\mathrm{d}}{\mathrm{d} t} \int_{\Omega}\left(\frac{\rho_{S}}{2}\left|u_{t}\right|^{2}+\frac{\delta_{i}}{2}\left|\mathcal{B} u_{t}\right|^{2}+\hat{K}_{R}(p)\right) \mathrm{d} x \\
& \quad+\int_{\Omega}\left(\left(\mathbf{B} \nabla_{s} u_{t}+P\left[\nabla_{s} u\right]\right): \nabla_{s} u_{t}+G[p]_{t} p+\frac{1}{\rho_{L}} \mu(p)|\nabla p|^{2}\right) \mathrm{d} x \\
& \quad=\int_{\Omega}\left(\beta Q_{R_{i}}(\theta) \operatorname{div} u_{t}+g \cdot u_{t}\right) \mathrm{d} x+\int_{\partial \Omega} \gamma_{p}(x) p\left(p^{*}-p\right) \mathrm{d} s(x) .
\end{aligned}
$$

By (1.3) and (2.29), we have $G[p]_{t} p \geq V_{G}[p]_{t}, V_{G}[p](x, t) \geq 0$, and $V_{G}[p](x, 0) \leq C|p(x, 0)|^{2} \leq C$ a. e. Integrating in time, taking into account (5.2) and the previous estimates, we get

$$
\left\|\nabla_{s} u_{t}^{(i)}\right\|_{2}+\left\|\nabla p^{(i)}\right\|_{2}+\delta_{i}\left|\mathcal{B} u_{t}^{(i)}(t)\right|_{2}+\int_{0}^{T} \int_{\partial \Omega} \gamma_{p}(x)\left|p^{(i)}\right|^{2} \mathrm{~d} s(x) \leq C
$$

for all $t \in(0, T)$. Consequently, as $\gamma_{p}$ does not identically vanish on $\partial \Omega$ by Hypothesis 3.1 , we also have

$$
\left\|p^{(i)}\right\|_{L^{2}\left(0, T ; W^{1,2}(\Omega)\right)} \leq C .
$$

\subsection{Estimate 8}

Test (4.4) by $\phi=u_{t t}=u_{t t}^{(i)}$. Then

$$
\begin{gathered}
\int_{\Omega}\left(\rho_{S}\left|u_{t t}\right|^{2}+\delta_{i}\left|\mathcal{B} u_{t t}\right|^{2}\right) \mathrm{d} x+\frac{1}{2} \frac{\mathrm{d}}{\mathrm{d} t} \int_{\Omega}\left(\mathbf{B} \nabla_{s} u_{t}+2 P\left[\nabla_{s} u\right]\right): \nabla_{s} u_{t} \mathrm{~d} x \\
\leq C \int_{\Omega}(|g|+|\nabla p|+|\nabla \theta|)\left|u_{t t}\right| \mathrm{d} x+\int_{\Omega} P\left[\nabla_{s} u\right]_{t}: \nabla_{s} u_{t} \mathrm{~d} x .
\end{gathered}
$$

From Proposition 2.1, (5.15), Korn's inequality, and (5.18) it follows that

$$
\left\|u_{t t}^{(i)}\right\|_{2}^{2}+\delta_{i}\left\|\mathcal{B} u_{t t}^{(i)}\right\|_{2}^{2}+\left|\nabla_{s} u_{t}^{(i)}(t)\right|_{2}^{2} \leq C
$$

for every $t \in(0, T)$. 


\section{6 $\quad$ Estimate 9}

We rewrite (4.4) in the form

$$
\int_{\Omega}\left(\left(\rho_{S} u_{t t}+\mathcal{B} u_{t}\right) \cdot \phi+\delta_{i} \mathcal{B} u_{t t} \cdot \mathcal{B} \phi\right) \mathrm{d} x=\int_{\Omega}(f+h) \cdot \phi \mathrm{d} x
$$

for all $\phi \in W^{2,2}\left(\Omega ; \mathbb{R}^{3}\right) \cap X_{0}$, where

$$
f=g-\beta \nabla Q_{R}(\theta)+\nabla p, \quad h=\operatorname{div} P\left[\nabla_{s} u\right] .
$$

We have $f \in L^{2}\left(\Omega ; \mathbb{R}^{3}\right)$ by (5.15), (5.18), and Hypothesis 3.1. To estimate $h$ in $L^{2}$, we use (2.12) and proceed as follows. Let $E_{l}, l=1,2,3$, be the $l$-th coordinate vector, let $(x, t) \in \Omega \times(0, T)$ be an arbitrary Lebesgue point of $\partial_{x_{l}} P\left[\nabla_{s} u\right]$, and let $s_{0} \in \mathbb{R}$ be sufficiently small such that $x+s E_{l} \in \Omega$ for $|s|<s_{0}$. By (2.12) and (2.14) we have

$$
\begin{gathered}
\left|P\left[\nabla_{s} u\right]\left(x+s E_{l}\right)-P\left[\nabla_{s} u\right](x, t)\right| \leq C\left(\left|\nabla_{s} u^{0}\left(x+s E_{l}\right)-\nabla_{s} u^{0}(x)\right|\right. \\
\left.+\int_{0}^{t}\left|\nabla_{s} u_{t}\left(x+s E_{l}, \tau\right)-\nabla_{s} u_{t}(x, \tau)\right| \mathrm{d} \tau\right),
\end{gathered}
$$

so that in the limit as $s \rightarrow 0$ we have

$$
\left|\frac{\partial}{\partial x_{l}} P\left[\nabla_{s} u\right](x, t)\right| \leq C\left(\left|\frac{\partial}{\partial x_{l}} \nabla_{s} u^{0}(x)\right|+\int_{0}^{t}\left|\frac{\partial}{\partial x_{l}} \nabla_{s} u_{t}(x, \tau)\right| \mathrm{d} \tau\right) \quad \text { a.e. }
$$

and

$$
|h(t)|_{2} \leq C\left(1+\int_{0}^{t}\left|\mathcal{B} u_{t}(\tau)\right| \mathrm{d} \tau\right)
$$

Consider now the Fourier expansion of $u=u^{(i)}$ in the form

$$
u(x, t)=\sum_{k=1}^{\infty} u_{k}(t) e_{k}(x)
$$

similar to (4.8), with coefficients

$$
u_{k}(t)=\int_{\Omega} u(t) \cdot e_{k}(x) \mathrm{d} x
$$

It follows e.g. from (5.18) that the series

$$
\mathcal{B} u_{t}(x, t)=\sum_{k=1}^{\infty} \lambda_{k} \dot{u}_{k}(t) e_{k}(x)
$$

is strongly convergent in $L^{2}\left(\Omega ; \mathbb{R}^{3}\right)$.

We now test (5.22) by $\phi=\mathcal{B} u_{t}^{(n)}$, where $u^{(n)}$ is as in (4.8) with coefficients $u_{k}(t)$ given by (5.28). Then

$$
\begin{aligned}
& \frac{\mathrm{d}}{\mathrm{d} t} \int_{\Omega}\left(\frac{\rho_{S}}{2} \mathbf{B} \nabla_{s} u_{t}^{(n)}: \nabla_{s} u_{t}^{(n)}+\frac{\delta_{i}}{2} \mathbf{B} \nabla_{s} \mathcal{B} u_{t}^{(n)}: \nabla_{s} \mathcal{B} u_{t}^{(n)}\right) \mathrm{d} x+\left|\mathcal{B} u_{t}^{(n)}(t)\right|_{2}^{2} \\
& \quad \leq C\left(1+|f(t)|_{2}+\int_{0}^{t}\left|\mathcal{B} u_{t}(\tau)\right|_{2} \mathrm{~d} \tau\right)\left|\mathcal{B} u_{t}^{(n)}(t)\right|_{2}
\end{aligned}
$$


hence,

$$
\begin{aligned}
& \frac{\mathrm{d}}{\mathrm{d} t} \int_{\Omega}\left(\frac{\rho_{S}}{2} \mathbf{B} \nabla_{s} u_{t}^{(n)}: \nabla_{s} u_{t}^{(n)}+\frac{\delta_{i}}{2} \mathbf{B} \nabla_{s} \mathcal{B} u_{t}^{(n)}: \nabla_{s} \mathcal{B} u_{t}^{(n)}\right) \mathrm{d} x+\left|\mathcal{B} u_{t}^{(n)}(t)\right|_{2}^{2} \\
& \quad \leq C\left(1+|f(t)|_{2}^{2}+\int_{0}^{t}\left|\mathcal{B} u_{t}(\tau)\right|_{2}^{2} \mathrm{~d} \tau\right)
\end{aligned}
$$

By (5.2), we can integrate this inequality from 0 to $t$, pass to the limit as $n \rightarrow \infty$, and use Gronwall's argument to obtain in particular that

$$
\left\|\mathcal{B} u_{t}\right\|_{2}=\left\|\mathcal{B} u_{t}^{(i)}\right\|_{2} \leq C .
$$

The next computation based on (4.17) is to check that

$$
\left\|u_{t}^{(i)}\right\|_{L^{r}\left(0, T ; C\left(\bar{\Omega} ; \mathbb{R}^{3}\right)\right)} \leq C \quad \text { for every } r \in[1,4) .
$$

Indeed, we choose any $\alpha \in[0,1 / 6)$ and put $\frac{1}{q}=\frac{1}{3}-\alpha$. By (5.21), (5.32), and (4.17) we have

$$
\left|\partial_{x_{j}} u_{t}^{(i)}(t)\right|_{q} \leq C\left(\left|\partial_{x_{j}} u_{t}^{(i)}(t)\right|_{2}+\left|\partial_{x_{j}} u_{t}^{(i)}(t)\right|_{2}^{1-\gamma}\left|\partial_{x_{j}} \nabla u_{t}^{(i)}(t)\right|_{2}^{\gamma}\right), \quad \gamma=\frac{\frac{1}{2}-\frac{1}{q}}{\frac{1}{3}}
$$

Then $\partial_{x_{j}} u_{t}^{(i)}$ is bounded in $L^{p}\left(0, T ; L^{q}\left(\Omega ; \mathbb{R}^{3}\right)\right)$ for $p \gamma=2$, that is,

$$
\left|\partial_{x_{j}} u_{t}^{(i)}\right|_{L^{p}\left(0, T ; L^{q}\left(\Omega ; \mathbb{R}^{3}\right)\right)} \leq C \text { for } q=\frac{3}{1-3 \alpha}, p=\frac{4}{6 \alpha+1} .
$$

By (15.21), $u_{t}^{(i)}$ is bounded in $L^{\infty}\left(0, T ; L^{2}\left(\Omega ; \mathbb{R}^{3}\right)\right)$, and by (4.17) for $\alpha>0$ we have

$$
\left|u_{t}^{(i)}(t)\right|_{\infty} \leq C\left(\left|u_{t}^{(i)}(t)\right|_{2}+\left|u_{t}^{(i)}(t)\right|_{2}^{1-\hat{\gamma}}\left|\nabla u_{t}^{(i)}(t)\right|_{q}^{\hat{\gamma}}\right), \quad \hat{\gamma}=\frac{\frac{1}{2}}{\frac{5}{6}-\frac{1}{q}} .
$$

We then obtain (5.33) for $r \hat{\gamma}=p$, that is,

$$
r=4 \frac{1+2 \alpha}{1+6 \alpha} \in[1,4) .
$$

\subsection{Estimate 10}

In this subsection, we prove the following statement.

Proposition 5.1. Let Hypothesis 3.1 hold. Then there exists a constant $C^{*}>0$ such that

$$
\left|p^{(i)}(x, t)\right| \leq C^{*} \quad \text { a. e. }
$$

Note that by (5.33), we have

$$
U \in L^{3}(0, T), \text { where we put } U(t):=1+\sup _{x \in \Omega}\left|u_{t}^{(i)}(x, t)\right| .
$$

As a preliminary step before we pass to the proof of Proposition [5.1, we prove the following auxiliary result for $p=p^{(i)}$. 
Lemma 5.2. There exist constants $c>0$ and $C>0$ independent of $m$ such that for every $m \geq 1$ and every $t \in[0, T]$ we have

$$
\begin{aligned}
& \int_{\Omega}|p(x, t)|^{2 m} \mathrm{~d} x+c \int_{0}^{t} \int_{\Omega}\left|\nabla\left(p|p|^{m-1}\right)\right|^{2} \mathrm{~d} x \mathrm{~d} \tau \\
& \leq C\left(1+m^{2}\right) \int_{0}^{t} U^{2}(\tau)\left(K^{2 m}+\int_{\Omega}|p(x, \tau)|^{2 m} \mathrm{~d} x\right) \mathrm{d} \tau .
\end{aligned}
$$

Proof. We choose an arbitrary $Q>0$ and $m \geq 1$, and test (4.5) by $h_{Q, m}(p)$, where we set

$$
h_{Q, m}(p)= \begin{cases}p|p|^{2 m} & \text { for }|p|<Q \\ Q^{2 m+1}+(2 m+1)(p-Q) Q^{2 m} & \text { for } p \geq Q \\ -Q^{2 m+1}+(2 m+1)(p+Q) Q^{2 m} & \text { for } p \leq-Q .\end{cases}
$$

We have $h_{Q, m}(p) \in L^{2}\left(0, T ; W^{1,2}(\Omega)\right)$ by virtue of (5.19), hence this is an admissible test function. By (2.35) we have $G_{0}[p]_{t} h_{Q, m}(p) \geq V_{h_{Q, m}}[p]_{t}$ and $K_{R}(p) p_{t} h_{Q, m}(p)=\hat{K}_{R, Q, m}(p)_{t}$, with $\hat{K}_{R, Q, m}(p)=\int_{0}^{p} K_{R}\left(p^{\prime}\right) h_{Q, m}\left(p^{\prime}\right) \mathrm{d} p^{\prime}$. We thus have

$$
\begin{gathered}
\frac{\mathrm{d}}{\mathrm{d} t} \int_{\Omega}\left(\hat{K}_{R, Q, m}(p)+V_{h_{Q, m}}[p]\right) \mathrm{d} x+\int_{\Omega} f^{\prime}(p) h_{Q, m}(p) p_{t} \mathrm{~d} x+\mu_{0}(2 m+1) \int_{\Omega}|\nabla p|^{2} \min \{|p|, Q\}^{2 m} \mathrm{~d} x \\
\leq-(2 m+1) \int_{\Omega} u_{t} \min \{|p|, Q\}^{2 m} \nabla p \mathrm{~d} x+\int_{\partial \Omega} \gamma(x)\left(p^{*}-p\right) h_{Q, m}(p) \mathrm{d} s(x)
\end{gathered}
$$

together with $\hat{K}_{R, Q, m}(p)(x, 0)=0$ by Hypothesis 2.4, and

$$
\int_{\Omega} V_{h_{Q, m}}[p](x, 0) \mathrm{d} x \leq C K^{2 m}
$$

as a consequence of (2.36) and (2.21), with $C$ independent of $Q$ and $m$. We estimate the right hand side of (5.41) as follows

$$
\begin{aligned}
& -(2 m+1) \int_{\Omega} u_{t} \min \{|p|, Q\}^{2 m} \nabla p \mathrm{~d} x \\
& \leq(2 m+1) U(t)\left(\int_{\Omega} \min \{|p|, Q\}^{2 m} \mathrm{~d} x\right)^{1 / 2}\left(\int_{\Omega}|\nabla p|^{2} \min \{|p|, Q\}^{2 m} \mathrm{~d} x\right)^{1 / 2} \\
& \leq \frac{\mu_{0}}{2}(2 m+1) \int_{\Omega}|\nabla p|^{2} \min \{|p|, Q\}^{2 m} \mathrm{~d} x+\frac{2 m+1}{2 \mu_{0}} U^{2}(t) \int_{\Omega} \min \{|p|, Q\}^{2 m} \mathrm{~d} x .
\end{aligned}
$$

For the boundary term, we have

$$
\int_{\partial \Omega} \gamma(x)\left(p^{*}-p\right) h_{Q, m}(p) \mathrm{d} s(x) \leq \frac{1}{2 m+2} \int_{\partial \Omega} \gamma(x)\left(\left|p^{*}\right|^{2 m+2}-\min \{|p|, Q\}^{2 m+2}\right) \mathrm{d} s(x) .
$$

On the left hand side of (5.41), we have

$$
\int_{0}^{t} f^{\prime}(p) h_{Q, m}(p) p_{t}(x, \tau) \mathrm{d} \tau=F_{Q, m}(p(x, t))-F_{Q, m}(p(x, 0)),
$$


where we set $F_{Q, m}(p)=\int_{0}^{p} f^{\prime}(z) h_{Q, m}(z) \mathrm{d} z$. We claim that for every $p \in \mathbb{R}$ we have

$$
\frac{f_{3}}{2 m+2}|p|^{2 m} \geq F_{Q, m}(p) \geq \frac{f_{2}}{4 m}\left(\min \{|p|, Q\}^{2 m}-1\right) .
$$

The upper bound is easy. We have for $z>0$ that $f^{\prime}(z) h_{Q, m}(z) \leq f_{3} z|z|^{2 m}$ and similarly for $z<0$, and it suffices to integrate. To get the lower bound, set

$$
\hat{F}_{Q, m}(p)=F_{Q, m}(p)-\frac{f_{2} \min \{|p|, Q\}^{2 m}}{4 m} .
$$

Then for $p>Q$ we have $\hat{F}_{Q, m}^{\prime}(p)=f^{\prime}(p) h_{Q, m}(p)>0$. For $p \in(0, Q)$ we have

$$
\hat{F}_{Q, m}^{\prime}(p)=f^{\prime}(p) p|p|^{2 m}-\frac{f_{2} p|p|^{2 m-1}}{2} \geq \frac{f_{2} p|p|^{2 m-1}}{2\left(1+p^{2}\right)}\left(p^{2}-1\right),
$$

hence the minimum of $\hat{F}_{Q, m}(p)$ is attained at $p=1$, with $\hat{F}_{Q, m}(1) \geq-\frac{f_{2}}{4 m}$, which is exactly (5.43). The case $p<0$ is symmetric.

Summarizing the above estimates, we obtain by integrating (5.41) from 0 to $t$ that

$$
\begin{aligned}
& \int_{\Omega} \min \{|p|, Q\}^{2 m}(x, t) \mathrm{d} x+\frac{\mu_{0}}{f_{2}} 2 m(2 m+1) \int_{0}^{t} \int_{\Omega}|\nabla p|^{2} \min \{|p|, Q\}^{2 m} \mathrm{~d} x \mathrm{~d} \tau \\
& \quad+\frac{2 m}{f_{2}(m+1)} \int_{\partial \Omega} \gamma(x) \min \{|p|, Q\}^{2 m+2} \mathrm{~d} s(x) \\
& \leq C\left(1+m^{2}\right) \int_{0}^{t} U^{2}(\tau)\left(K^{2 m}+\int_{\Omega} \min \{|p|, Q\}^{2 m}(x, \tau) \mathrm{d} x\right) \mathrm{d} \tau .
\end{aligned}
$$

In particular, the function $w_{m}(t):=\int_{\Omega} \min \{|p|, Q\}^{2 m}(x, t) \mathrm{d} x$ satisfies the inequality

$$
w_{m}(t) \leq C\left(1+m^{2}\right) \int_{0}^{t} U^{2}(\tau)\left(K^{2 m}+w_{m}(\tau)\right) \mathrm{d} \tau
$$

and by Gronwall's argument (note that $U^{2} \in L^{1}(0, T)$ by (5.38) ), there exists a constant $C(m)$ depending on $m$ and independent of $Q$ such that $\sup _{t \in[0, T]} w_{m}(t) \leq C(m)$. Hence, we can let $Q$ tend to $\infty$ in (5.44) and obtain

$$
\begin{aligned}
& \int_{\Omega}|p|^{2 m}(x, t) \mathrm{d} x+\frac{\mu_{0}}{f_{2}} 2 m(2 m+1) \int_{0}^{t} \int_{\Omega}|\nabla p|^{2}|p|^{2 m} \mathrm{~d} x \mathrm{~d} \tau \\
& \quad+\frac{2 m}{f_{2}(m+1)} \int_{\partial \Omega} \gamma(x)|p|^{2 m+2} \mathrm{~d} s(x) \\
& \leq C\left(1+m^{2}\right) \int_{0}^{t} U^{2}(\tau)\left(K^{2 m}+\int_{\Omega}|p|^{2 m}(x, \tau) \mathrm{d} x\right) \mathrm{d} \tau .
\end{aligned}
$$

In particular,

$$
p \in L^{\infty}\left(0, T ; L^{q}(\Omega)\right) \quad \forall q \geq 1
$$


but the norm of $p$ in this space still depends on $q$. Note that

$$
\begin{aligned}
\int_{0}^{t} \int_{\Omega}|\nabla p|^{2}|p|^{2 m} \mathrm{~d} x \mathrm{~d} \tau & \geq \int_{0}^{t} \int_{\Omega}|\nabla p|^{2}|p|^{2 m-2} \mathrm{~d} x \mathrm{~d} \tau-\int_{0}^{t} \int_{\Omega}|\nabla p|^{2} \mathrm{~d} x \mathrm{~d} \tau \\
& \geq \int_{0}^{t} \int_{\Omega}|\nabla p|^{2}|p|^{2 m-2} \mathrm{~d} x \mathrm{~d} \tau-C
\end{aligned}
$$

by virtue of (5.18). Indeed, it suffices to split the integration domain into the parts where $p \geq 1$ and $p<1$. Using (5.47), we rewrite (5.44) as

$$
\begin{aligned}
& \int_{\Omega}|p(x, t)|^{2 m} \mathrm{~d} x+\frac{\mu_{0}}{f_{2}} \frac{2(2 m+1)}{m} \int_{0}^{t} \int_{\Omega}\left|\nabla\left(p|p|^{m-1}\right)\right|^{2} \mathrm{~d} x \mathrm{~d} \tau+\frac{2 m}{f_{2}(m+1)} \int_{\partial \Omega} \gamma(x)|p|^{2 m+2} \mathrm{~d} s(x) \\
& \leq C\left(1+m^{2}\right) \int_{0}^{t} U^{2}(\tau)\left(K^{2 m}+\int_{\Omega}|p(x, \tau)|^{2 m} \mathrm{~d} x\right) \mathrm{d} \tau .
\end{aligned}
$$

Putting $c:=4 \mu_{0} / f_{2}$, we obtain (5.39), and Lemma 5.2 is proved.

Proof of Proposition 5.1. Set $w:=p|p|^{m-1}$ for $p=p^{(i)}$. Then (5.39) reads

$$
|w(t)|_{2}^{2}+c \int_{0}^{t}|\nabla w(\tau)|_{2}^{2} \mathrm{~d} \tau \leq C\left(1+m^{2}\right) \int_{0}^{t} U^{2}(\tau)\left(K^{2 m}+|w(\tau)|_{2}^{2}\right) \mathrm{d} \tau .
$$

We now choose $s=3 / 4$, and invoke the Gagliardo-Nirenberg inequality (4.17) in the form

$$
|w(\tau)|_{2} \leq C\left(|w(\tau)|_{2 s}+|w(\tau)|_{2 s}^{1-\gamma}|\nabla w(\tau)|_{2}^{\gamma}\right), \quad \gamma=\frac{\frac{1}{2 s}-\frac{1}{2}}{\frac{1}{2 s}-\frac{1}{6}}=\frac{1}{3} .
$$

Hölder's inequality enables us to rewrite (5.49) as

$$
\begin{aligned}
& |w(t)|_{2}^{2}+c \int_{0}^{t}|\nabla w(\tau)|_{2}^{2} \mathrm{~d} \tau \\
& \leq C\left(1+m^{2}\right) \int_{0}^{t} U^{2}(\tau)\left(K^{2 m}+|w(\tau)|_{2 s}^{2}\right) \mathrm{d} \tau+C\left(1+m^{2}\right) \int_{0}^{t} U^{2}(\tau)|w(\tau)|_{2 s}^{4 / 3}|\nabla w(\tau)|_{2}^{2 / 3} \mathrm{~d} \tau \\
& \leq C\left(1+m^{2}\right) \int_{0}^{t} U^{2}(\tau)\left(K^{2 m}+|w(\tau)|_{2 s}^{2}\right) \mathrm{d} \tau \\
& \quad+C\left(1+m^{2}\right)\left(\int_{0}^{t} U^{3}(\tau)|w(\tau)|_{2 s}^{2} \mathrm{~d} \tau\right)^{2 / 3}\left(\int_{0}^{t}|\nabla w(\tau)|_{2}^{2} \mathrm{~d} \tau\right)^{1 / 3},
\end{aligned}
$$

and we conclude that

$$
|w(t)|_{2}^{2} \leq C\left(1+m^{3}\right) \int_{0}^{t} U^{3}(\tau)\left(K^{2 m}+|w(\tau)|_{2 s}^{2}\right) \mathrm{d} \tau,
$$

or, in terms of $p$,

$$
|p(t)|_{2 m}^{2 m} \leq C\left(1+m^{3}\right) \int_{0}^{t} U^{3}(\tau)\left(K^{2 m}+|p(\tau)|_{2 s m}^{2 m}\right) \mathrm{d} \tau .
$$


We have $U \in L^{3}(0, T)$ by (5.38). If now $V$ is a constant such that $\max \left\{K,|p(\tau)|_{2 s m}\right\} \leq V$, then

$$
\tilde{V}:=\max \left\{K,|p(t)|_{2 m}\right\} \leq\left(C\left(1+m^{3}\right)\right)^{1 / 2 m} V .
$$

We now define $m_{k}:=s^{-k}=(4 / 3)^{k}$, and put

$$
V_{k}:=\max \left\{K, \sup _{\tau \in(0, T)}|p(\tau)|_{2 m_{k}}\right\} .
$$

By (5.52) we have for all $k=2,3, \ldots$ that

$$
V_{k} \leq\left(C\left(1+\left(\frac{4}{3}\right)^{3 k}\right)\right)^{\left((3 / 4)^{k}\right) / 2} V_{k-1}
$$

hence

$$
\log V_{k}-\log V_{k-1} \leq \frac{1}{2}\left(\frac{3}{4}\right)^{k} \log \left(C\left(1+\left(\frac{4}{3}\right)^{3 k}\right)\right)
$$

The right hand side of (5.54) is a convergent series, and $V_{1}$ is finite by virtue of (5.46), so that we can conclude that the sequence $\left\{V_{k}\right\}$ is uniformly bounded independently of $i$, which we wanted to prove.

\subsection{Estimate 11}

Test (4.5) by $\psi=M(p)_{t}$, with $M(p)$ given by (3.6). From (2.24) and Proposition 5.1, it follows that there exists a constant $c>0$ such that for every $t \in(0, T)$ we have

$$
\begin{aligned}
c \int_{\Omega} p_{t}^{2}(x, t) \mathrm{d} x+\frac{1}{2 \rho_{L}} \frac{\mathrm{d}}{\mathrm{d} t} \int_{\Omega}|\nabla M(p)|^{2}(x, t) \mathrm{d} x+\frac{\mathrm{d}}{\mathrm{d} t} \int_{\partial \Omega} \gamma_{p}(x) \hat{M}(p)(x, t) \mathrm{d} s(x) \\
\leq \int_{\Omega}\left(\left|\operatorname{div} u_{t}\right|\left|M(p)_{t}\right|\right)(x, t) \mathrm{d} x+\frac{\mathrm{d}}{\mathrm{d} t} \int_{\partial \Omega} \gamma_{p}(x)\left(M(p) p^{*}\right)(x, t) \mathrm{d} s(x) \\
\quad-\int_{\partial \Omega} \gamma_{p}(x)\left(M(p) p_{t}^{*}\right)(x, t) \mathrm{d} s(x)
\end{aligned}
$$

with $\hat{M}(p)=\int_{0}^{p} p^{\prime} \mu\left(p^{\prime}\right) \mathrm{d} p^{\prime}$. Hence, using also (5.21), we have for all $t \in(0, T)$ that

$$
\left\|p_{t}^{(i)}\right\|_{2}^{2}+\left|\nabla p^{(i)}(t)\right|_{2}^{2}+\int_{\partial \Omega} \gamma_{p}(x)\left|p^{(i)}\right|^{2}(x, t) \mathrm{d} s(x) \leq C .
$$

By comparison in Eq. (4.5), we also obtain that

$$
\left\|M\left(p^{(i)}\right)\right\|_{L^{2}\left(0, T ; W^{2,2}(\Omega)\right)} \leq C .
$$




\subsection{Estimate 12}

By the same argument based on (5.56) -(5.57), we have similarly as in (5.35) that

$$
\left\|\nabla p^{(i)}\right\|_{L^{p}\left(0, T ; L^{q}(\Omega)\right)} \leq C \text { for } q=\frac{3}{1-3 \alpha}, p=\frac{4}{6 \alpha+1}
$$

for every $\alpha \in[0,1 / 6)$. In particular, for $\alpha=1 / 30$, we have $\partial_{x_{i}} u_{t}, \partial_{x_{i}} p \in L^{10 / 3}(\Omega \times(0, T))$. Hence, Eq. (4.6) has the form

$$
\int_{\Omega}\left(c_{0} \theta_{t} \zeta+\kappa(\theta) \nabla \theta \cdot \nabla \zeta\right) \mathrm{d} x+\int_{\partial \Omega} \gamma_{\theta}(x)\left(\theta-\theta^{*}\right) \zeta \mathrm{d} s(x)=\int_{\Omega}(A(x, t)+B(x, t) \theta) \zeta \mathrm{d} x
$$

with $A \in L^{5 / 3}(\Omega \times(0, T)), B \in L^{10 / 3}(\Omega \times(0, T))$ bounded independently of $i$. We test (5.59) with $\zeta=\theta^{r}$ (with $r$ to be chosen later) and obtain using Hölder's inequality for every $t \in(0, T)$ that

$$
\begin{aligned}
& \frac{1}{r+1} \int_{\Omega} \theta^{r+1}(x, t) \mathrm{d} x+r \int_{0}^{T} \int_{\Omega} \theta^{r-1} \kappa(\theta)|\nabla \theta|^{2}(x, \tau) \mathrm{d} x \mathrm{~d} \tau+\int_{0}^{T} \int_{\partial \Omega} \gamma_{\theta}(x) \theta^{r+1}(x, \tau) \mathrm{d} s(x) \mathrm{d} \tau \\
& \quad \leq C\left(1+\|\theta\|_{5 r / 2}^{r}+\|\theta\|_{10(r+1) / 7}^{r+1}\right)
\end{aligned}
$$

with, by Hypothesis 3.1 ((ii),

$$
\theta^{r-1} \kappa(\theta)|\nabla \theta|^{2} \geq \frac{1}{C} \theta^{r+a}|\nabla \theta|^{2}
$$

We already have the estimate (5.16). Assume that for some $z \geq 8 / 3$ we have proved

$$
\|\theta\|_{z} \leq C_{0}(z)
$$

with some $C_{0}(z)>0$. For this value of $z$ we choose in (5.60)

$$
r=r(z)= \begin{cases}(7 z / 10)-1 & \text { for } z \in[8 / 3,10 / 3] \\ 2 z / 5 & \text { for } z>10 / 3\end{cases}
$$

Then $\|\theta\|_{5 r / 2}^{r}+\|\theta\|_{10(r+1) / 7}^{r+1} \leq C\left(1+\|\theta\|_{z}^{r+1}\right)$, and we have by virtue of (5.60) $-(5.61)$ that

$$
\frac{1}{r+1} \int_{\Omega} \theta^{r+1}(x, t) \mathrm{d} x+r \int_{0}^{T} \int_{\Omega} \theta^{r+a}|\nabla \theta|^{2}(x, \tau) \mathrm{d} x \mathrm{~d} \tau \leq C\left(1+C_{0}(z)^{r+1}\right) .
$$

Set

$$
p=\frac{r+a}{2}+1, s=\frac{r+1}{p}, w=\theta^{p} .
$$

Then (5.64) can be written as

$$
\frac{1}{r+1} \int_{\Omega} w^{s}(x, t) \mathrm{d} x+r \int_{0}^{T} \int_{\Omega}|\nabla w|^{2}(x, \tau) \mathrm{d} x \mathrm{~d} \tau \leq C\left(1+C_{0}(z)^{r+1}\right) .
$$


For $s<q<6$ we have by virtue of the Gagliardo-Nirenberg inequality (4.17) that

$$
|w(\tau)|_{q} \leq C\left(|w(\tau)|_{s}+|w(\tau)|_{s}^{1-\gamma}|\nabla w(\tau)|_{2}^{\gamma}\right), \quad \gamma=\frac{\frac{1}{s}-\frac{1}{q}}{\frac{1}{s}-\frac{1}{6}}
$$

If $q$ is chosen in such a way that $q \gamma=2$, that is,

$$
q=\frac{2}{3} s+2
$$

then it follows from (5.67) and Young's inequality that

$$
\|w\|_{q} \leq C\left(\sup _{\tau \in[0, T]}|w(\tau)|_{s}+\sup _{\tau \in[0, T]}|w(\tau)|_{s}^{1-\gamma}\|\nabla w\|_{2}^{2 / q}\right) \leq C\left(\sup _{\tau \in[0, T]}|w(\tau)|_{s}+\|\nabla w\|_{2}\right)
$$

By (5.66), we have

$$
\begin{aligned}
\sup _{\tau \in[0, T]}|w(\tau)|_{s} & \leq C\left((1+r)\left(1+C_{0}(z)^{r+1}\right)\right)^{1 / s} \\
\|\nabla w\|_{2} & \leq C\left(1+C_{0}(z)^{r+1}\right)^{1 / 2} \leq C\left(1+C_{0}(z)^{r+1}\right)^{1 / s}
\end{aligned}
$$

(note that $s<2$ ), so that

$$
\|w\|_{q} \leq C\left((1+r)\left(1+C_{0}(z)^{r+1}\right)\right)^{1 / s}
$$

Putting

$$
\hat{z}=p q=\frac{5}{3} r(z)+\frac{8}{3}+a
$$

we have by $(5.70)$ that

$$
\|\theta\|_{\hat{z}}=\|w\|_{q}^{1 / p} \leq C\left((1+r)\left(1+C_{0}(z)^{r+1}\right)\right)^{1 /(p s)},
$$

and using the identity $p s=r+1$, we obtain the implication

$$
\|\theta\|_{z} \leq C_{0}(z) \Longrightarrow\|\theta\|_{\hat{z}} \leq \hat{C}_{0}(z), \quad \hat{C}_{0}(z)=C\left(1+C_{0}(z)\right) .
$$

The sequence

$$
z_{k}=\frac{5}{3} r\left(z_{k-1}\right)+\frac{8}{3}+a, \quad z_{0}=\frac{8}{3}
$$

converges to $z_{\infty}=8+3 a$. After finitely many iterations we obtain

$$
\|\theta\|_{z} \leq C \text { for every } z<8+3 a .
$$

Consequently, by (5.60) -(5.64), we have for $r<\frac{16}{5}+\frac{6}{5}$ and $t \in(0, T)$ that

$$
\int_{\Omega} \theta^{r+1}(x, t) \mathrm{d} x+r \int_{0}^{T} \int_{\Omega} \theta^{r-1} \kappa(\theta)|\nabla \theta|^{2}(x, \tau) \mathrm{d} x \mathrm{~d} \tau+\int_{0}^{T} \int_{\partial \Omega} \gamma_{\theta} \theta^{r+1}(x, \tau) \mathrm{d} s(x) \mathrm{d} \tau \leq C .
$$


This enables us to derive an upper bound for the integral $\int_{\Omega} \kappa(\theta) \nabla \theta \cdot \nabla \zeta \mathrm{d} x$ which we need for getting an estimate for $\theta_{t}$ from the equation (5.59). We have by Hölder's inequality and Hypothesis 3.1(ii) that

$$
\begin{aligned}
\int_{\Omega}|\kappa(\theta) \nabla \theta \cdot \nabla \zeta| \mathrm{d} x & =\int_{\Omega}\left|\theta^{(1-r) / 2} \kappa^{1 / 2}(\theta) \theta^{(r-1) / 2} \kappa^{1 / 2}(\theta) \nabla \theta \cdot \nabla \zeta\right| \mathrm{d} x \\
& \leq C\left(\int_{\Omega} \theta^{r-1} \kappa(\theta)|\nabla \theta|^{2} \mathrm{~d} x\right)^{1 / 2}\left(\int_{\Omega} \theta^{2+b-r}|\nabla \zeta|^{2} \mathrm{~d} x\right)^{1 / 2} .
\end{aligned}
$$

We now want to choose $\eta>0$ and $r$ satisfying (5.74) such that

$$
2+b-r=\frac{r+1}{1+\eta}
$$

or, equivalently,

$$
r=\frac{(1+2 \eta)+b(1+\eta)}{2+\eta}=\frac{1+b}{2}+\frac{\eta(b+3)}{2(2+\eta)} .
$$

If $b$ satisfies the condition in Hypothesis 3.1(ii), then there exists $\eta \in(0,1)$ such that for $r$ given by (5.77) the condition in (5.74) holds. Hence, by (5.75),

$$
\int_{\Omega}|\kappa(\theta) \nabla \theta \cdot \nabla \zeta| \mathrm{d} x \leq C\left(\int_{\Omega} \theta^{r-1} \kappa(\theta)|\nabla \theta|^{2} \mathrm{~d} x\right)^{1 / 2}\left(\int_{\Omega} \theta^{r+1} \mathrm{~d} x\right)^{1 /(1+\eta)}\left(\int_{\Omega}|\nabla \zeta|^{q^{*}} \mathrm{~d} x\right)^{1 / q^{*}}
$$

with $q^{*}=\frac{2(1+\eta)}{\eta}$. Hence, by (5.74)

$$
\int_{0}^{T} \int_{\Omega}\left|\kappa\left(\theta^{(i)}\right) \nabla \theta^{(i)} \cdot \nabla \zeta\right| \mathrm{d} x \mathrm{~d} t \leq C\|\zeta\|_{L^{2}\left(0, T ; W^{1, q^{*}}(\Omega)\right)}
$$

By (5.35) and (5.58) for $\alpha=0$, the functions $A$ and $B$ in (5.59) satisfy uniform bounds $A \in L^{2}\left(0, T ; L^{3 / 2}(\Omega)\right), B \in L^{4}\left(0, T ; L^{3}(\Omega)\right)$ independent of $i$, so that testing with $\zeta \in$ $L^{2}\left(0, T ; W^{1, q^{*}}(\Omega)\right)$ is admissible. We thus obtain from (5.59) that

$$
\int_{0}^{T} \int_{\Omega} \theta_{t}^{(i)} \zeta \mathrm{d} x \mathrm{~d} t \leq C\|\zeta\|_{L^{2}\left(0, T ; W^{1, q^{*}}(\Omega)\right)}
$$

\subsection{Passage to the limit as $i \rightarrow \infty$}

In the system (4.4)-(4.6) with $\delta=\delta_{i}, R=R_{i}$, and $(u, p, \theta)=\left(u^{(i)}, p^{(i)}, \theta^{(i)}\right)$, we fix test functions $\phi \in W^{2,2}\left(\Omega ; \mathbb{R}^{3}\right) \cap X_{0}, \psi \in X$, and $\zeta \in L^{2}\left(0, T ; W^{1, q^{*}}(\Omega)\right)$ with $q^{*}$ from (5.79). The term $\delta_{i} \mathcal{B} u_{t t}^{(i)}$ in (4.4) converges to 0 in $L^{2}$ by (5.21), the regularization $K_{R_{i}}(p)$ vanishes for $R_{i}>C^{*}$ by Proposition 5.1.

By (5.21) and (5.32), the sequence $\left\{\nabla_{s} u_{t}^{(i)}\right\}$ is precompact in $L^{4}\left(0, T ; L^{q}\left(\Omega ; \mathbb{R}_{\text {sym }}^{3 \times 3}\right)\right)$ for every $q \in[1,3)$. Similarly, by (5.56) $-(5.57),\left\{\nabla p^{(i)}\right\}$ is precompact in $L^{4}\left(0, T ; L^{q}\left(\Omega ; \mathbb{R}^{3}\right)\right)$ for every $q \in[1,3)$, and $\left\{p^{(i)}\right\}$ is precompact in $L^{q}(\Omega ; C[0, T])$ for every $q>1$ by virtue of Proposition 5.1. Finally, by (5.15), (5.73), and (5.80), $\left\{\theta^{(i)}\right\}$ is precompact e.g. in $L^{8}(\Omega \times(0, T))$. Hence, 
using also (5.79), we select a subsequence and pass to the weak limit in the linear terms in (4.4)(4.6), and to the strong limit in all nonlinear non-hysteretic terms. Obviously, if $\theta^{(i)}$ converge strongly to $\theta$ in $L^{8}(\Omega \times(0, T))$, then $Q_{R_{i}}\left(\theta^{(i)}\right) \rightarrow \theta$ strongly in $L^{8}(\Omega \times(0, T))$ as well, and if $\left|\nabla p^{(i)}\right|^{2} \rightarrow|\nabla p|^{2}$ in $L^{2}\left(0, T ; L^{q / 2}(\Omega)\right)$ strongly, then $Q_{R_{i}}\left(\left|\nabla p^{(i)}\right|^{2}\right) \rightarrow|\nabla p|^{2}$ in $L^{2}\left(0, T ; L^{q / 2}(\Omega)\right)$ strongly. By the same argument as in Subsection 4.5, we show that the hysteresis terms $G\left[p^{(i)}\right]_{t}$, $\left\|D_{P}\left[\nabla_{s} u^{(i)}\right]_{t}\right\|_{*}, \quad\left|D_{G}\left[p^{(i)}\right]_{t}\right|$ converge weakly in $L^{2}(\Omega \times(0, T))$, and that the limit as $i \rightarrow \infty$ yields a solution to (3.2) -(3.4) with $\phi \in W^{2,2}\left(\Omega ; \mathbb{R}^{3}\right) \cap X_{0}$. By density we conclude that $\phi \in X_{0}$ is an admissible test function, which completes the proof of Theorem 3.2 .

\section{References}

[1] Albers B. Modeling the hysteretic behavior of the capillary pressure in partially saturated porous media - a review. Acta Mechanica. 2014; 225 (8): 2163-2189.

[2] Albers B. On the influence of the hysteretic behavior of the capillary pressure on the wave propagation in partially saturated soils. Submitted to Journal of Physics: Conference Series, Proceedings of the 7th International Workshop on Multi-Rate Processes and Hysteresis. 2014.

[3] Albers B. Main drying and wetting curves of soils - on measurements, prediction and influence on wave propagation. Engineering Transactions. 2015; 63 (1): 5-34.

[4] Albers B. Modeling and Numerical Analysis of Wave Propagation in Saturated and Partially Saturated Porous Media. Habilitation Thesis. Veröffentlichungen des Grundbauinstitutes der Technischen Universität Berlin, Shaker: Aachen; 2010.

[5] Albers B, Krejčí P. Unsaturated porous media flow with thermomechanical interaction. Math. Meth. Appl. Sci., DOI: 10.1002/mma.3635, 2015.

[6] Bagagiolo F, Visintin A. Hysteresis in filtration through porous media. Z. Anal. Anwendungen. 2000; 19 (4): 977-997.

[7] Bagagiolo F, Visintin A. Porous media filtration with hysteresis. Adv. Math. Sci. Appl. 2004; 14 (2): 379-403.

[8] Besov OV, Il'in VP, Nikol'skiu SM. Integral Representations of Functions and Imbedding Theorems. Scripta Series in Mathematics. Halsted Press (John Wiley \& Sons): New York-Toronto, Ont.London; 1978 (Vol. I), 1979 (Vol. II). Russian version Nauka: Moscow; 1975.

[9] Feireisl E., Petzeltová H., Rocca E. Existence of solutions to some models of phase changes with microscopic movements. Math. Meth. Appl. Sci. 2009; 32: 1345-1369.

[10] Flynn D. Modelling the flow of water through multiphase porous media with the Preisach model. PhD Thesis. University College Cork; 2008.

[11] Flynn D, McNamara H, O'Kane JP, Pokrovskiu AV. Application of the Preisach model to soilmoisture hysteresis. The Science of Hysteresis, Volume 3, Eds. Bertotti G, Mayergoyz I. Academic Press: Oxford; 2006: 689-744.

[12] Gilbarg D, Trudinger NS. Elliptic Partial Differential Equations of Second Order. Springer-Verlag: Berlin-Heidelberg-New York-Tokyo; 1983. 
[13] Haverkamp R, Reggiani P, Ross PJ, Parlange J-Y. Soil water hysteresis prediction model based on theory and geometric scaling. Environmental Mechanics, Water, Mass and Engergy Transfer in the Biosphere. Eds. Raats PAC, Smiles D, Warrick AW. American Geophysical Union: 2002; 213-246.

[14] Krasnosel'skiı̆ MA, Pokrovskiı̌ AV. Systems with Hysteresis. Springer: Berlin; 1989. Russian edition: Nauka: Moscow; 1983.

[15] Krejčí P. Hysteresis, Convexity and Dissipation in Hyperbolic Equations. Gakuto Intern. Ser. Math. Sci. Appl. Vol. 8. Gakkotōsho: Tokyo; 1996.

[16] Krejčí P. On Maxwell equations with the Preisach hysteresis operator: the one-dimensional timeperiodic case. Apl. Mat. 1989; 34: 364-374.

[17] Krejčí P. The Preisach hysteresis model: Error bounds for numerical identification and inversion. Discrete Cont. Dyn. Systems Series S. 2013; 6: 101-119.

[18] Lemaitre J, Chaboche J-L. Mechanics of Solid Materials. Cambridge University Press: Cambridge; 1990.

[19] Nečas J. Les méthodes directes en théorie des équations elliptiques. Academia: Prague; 1967.

[20] Preisach F. Über die magnetische Nachwirkung. Z. Phys. 1935; 94: 277-302 (in German).

[21] Rocca E, Rossi R. "Entropic" solutions to a thermodynamically consistent PDE system for phase transitions and damage. SIAM J. Math. Anal. 2015; 47: 2519-2586.

[22] Showalter RE. Diffusion in deforming porous media. Dyn. Contin. Discrete Impuls. Syst. Ser. A Math. Anal. 2003; 10 (5): 661-678.

[23] Showalter RE, Stefanelli U. Diffusion in poro-plastic media. Math. Methods Appl. Sci. 2004; 27: 2131-2151. 